\title{
New Model of Retinocollicular Mapping Predicts the Mechanisms of Axonal Competition and Explains the Role of Reverse Molecular Signaling during Development
}

\author{
François Grimbert and Jianhua Cang \\ Department of Neurobiology, Northwestern University, Evanston, Illinois 60208
}

Precise connections in the brain result from elaborate processes during development. In the visual system, axonal projections from retinal ganglion cells (RGCs) onto the superior colliculus form a precise retinotopic map. Studies have revealed that the development of retinocollicular maps involves three main factors: graded expression of molecular guidance cues such as EphAs and ephrin-As, activitydependent processes driven by spontaneous activity in RGCs, and different forms of axonal competition. In this study, we developed a new, versatile model including these factors. We first modeled the selective arborization of RGC axons, mediated by EphA/ephrin-A signaling, without assuming that this initial process instructed the map's final topology. We also derived an integro-differential equation modeling a second, dynamic phase in which activity-dependent plasticity of axonal arbors combined with their competition for collicular resources can deeply remodel the topology of immature maps. Our model hence challenges the view that retinotopic maps are instructed by matching molecular gradients and then merely refined by activity-dependent processes. We reproduce fine features of retinotopic map development in wild-type and various transgenic mice, allowing a new understanding of the underlying mechanisms. Our model predicts that competition is not based on comparisons of axonal EphA receptor levels but rather relies on the optimization of collicular resources mediated by neurotrophic receptors such as $\mathrm{p} 75^{\mathrm{NTR}}$. Our model finally clarifies the elusive role of reverse signaling between retinal ephrin-As and collicular EphAs by reproducing for the first time the phenotypes of two mouse genotypes in which this function is altered.

\section{Introduction}

Neuronal projections in the visual system are precisely organized to preserve a continuous retinotopic representation of the space, which is presumably required for normal visual perception (Chklovskii and Koulakov, 2004). How retinotopic maps are established during development is thus an important question and has been extensively studied in the maps made by retinal ganglion cells (RGCs) onto the superior colliculus (SC) in mice and its homolog, the optic tectum, in lower vertebrates.

These studies revealed that molecular cues are critical in mapping the retinocollicular projections. The EphA family of receptor tyrosine kinases and their ligands, ephrin-As, are expressed in gradients in the retina and the SC, respectively (McLaughlin and O'Leary, 2005). Their interaction, known as forward signaling, is repulsive, so that temporal RGCs (high EphAs) project to the anterior SC (low ephrin-As). In addition, opposing gradients of ephrin-As and EphAs are found in the retina and SC, and ephrin-

\footnotetext{
Received December 13, 2011; revised May 18, 2012; accepted May 23, 2012.

Author contributions: F.G. and J.C. designed research; F.G. performed research; F.G. analyzed data; F.G. and J.C. wrote the paper.

This work was supported by National Institutes of Health Grants EY018621 and EY020950, a Sloan Research Fellowship, a Klingenstein Fellowship Award in Neurosciences, and a Brain Research Foundation Seed Grant (J.C.). We thank Lupeng Wang and Xinyu Zhao for helpful discussions and comments.

Correspondence should be addressed to either François Grimbert or Jianhua Cang, Department of Neurobiology, Northwestern University, 2205 Tech Drive, Hogan 2-160, Evanston, IL 60208, E-mail: francois.grimbert@gmail.com or cang@northwestern.edu.

DOI:10.1523/JNEUROSCI.6180-11.2012

Copyright $\odot 2012$ the authors $\quad 0270-6474 / 12 / 329755-14 \$ 15.00 / 0$
}

expressing RGC axons can respond to collicular EphAs acting as ligands, hence mediating repulsion through reverse signaling. While reverse signaling is required in normal retinocollicular mapping, its function remains elusive (Rashid et al., 2005; Lim et al., 2008). Activity-dependent processes, driven by spontaneous RGC activity in the form of retinal waves, and axonal competition also play important roles in retinocollicular development (Ruthazer and Cline, 2004). In lower vertebrates, axons preferentially extend in sparsely innervated areas of the optic tectum (Ruthazer et al., 2003), and ablation experiments show that RGC axons from incomplete retinas invade the whole tectum (Udin and Gaze, 1983). Although these findings suggest a form of competition between RGC axons during map formation, the function of competition in retinocollicular mapping and its underlying mechanisms are not well understood.

A number of computational models have been developed in the past 40 years in order to understand retinocollicular/retinotectal map formation quantitatively (Goodhill and $\mathrm{Xu}, 2005$; Simpson et al., 2009). Although taking different forms, these models have largely assumed an instructive role for molecular cues, in which positional information is encoded by matching EphA/ephrin-A expression levels between RGC axons and their collicular targets or by sorting RGC axons based on their molecular expression levels. A few models have also included a form of Hebbian plasticity, essentially as a refining agent improving the precision of the coarse map established by the molecular gradients (Yates et al., 2004; Tsigankov and Koulakov, 2006; Godfrey et al., 2009). Finally, several models have featured axonal compe- 
tition but rarely provided new insights on its possible mechanisms (Simpson et al., 2009). In this study, we present an alternative model featuring "permissive" molecular signaling and a combination of Hebbian plasticity and axonal competition that actively shape local retinotopy. Within this new framework, we predict that axonal competition is not based on comparisons of EphA receptor levels and that p75 neurotrophic receptors are involved in competitive resource optimization. Finally, we clarify the role of reverse signaling by reproducing the developmental features of two lines of mice in which this function is impaired.

\section{Materials and Methods}

Arborization phase. In this section we review the technicalities of our arborization model. The modeling assumptions and the biological interpretation of our approach are detailed in the Results. In the following, retinal coordinates (nasotemporal, $x_{N T}$; dorsoventral, $x_{D V}$ ) and collicular coordinates (anteroposterior, $y_{A P}$; lateromedial, $y_{L M}$ ) are expressed as a percentage along retinal or collicular axes.

The retina is modeled as an $N_{r} \times N_{r}$ array of RGCs $\left(N_{r}=100\right)$. At the arborization phase, each RGC first establishes a pool of $n_{p}$ potential axonal arbors in the SC, with $n_{p} \geq n_{a}\left(n_{p}=7, n_{a}=3\right)$, and then only retains the $n_{a}$ most appropriate arbors through a noisy selection process $\left(n_{a}=3\right.$ accounts reasonably well for RGC arborizations while keeping computation times in the dynamic phase short). Arborization is based on a two-variable function $P_{A}(x, y)$ depending on an RGC's position along the nasotemporal (N-T) axis $(x)$ and a potential position of one of its arbors along the anteroposterior (A-P) axis $(y) . P_{A}(x, y)$ is the probability that the arbor develops, considering EphA and ephrin-A expression levels at positions $x$ and $y . P_{A}=P_{F} \times P_{R}$, where $P_{F}$ and $P_{R}$ model the inhibitory contributions of forward and reverse signaling.

We first calculate, for each RGC, the collicular coordinates of its $n_{p}$ potential arbors $\left(y_{L M}\right.$ and $\left.y_{A P}\right)$. The lateromedial (L-M) arbor positions are computed according to a very basic stochastic model. In the case of a uniform retinotopic mapping of the dorsoventral (D-V) axis of the retina onto the collicular L-M axis, $y_{L M}$ equals $x_{D V}$. In the model, we add L-M errors to account for the imperfect initial arborizations observed experimentally (Hindges et al., 2002). So, $y_{L M}=x_{D V}+E_{L M}+e_{L M}$. $E_{L M}$ is drawn randomly for each RGC and is the same for all its arbors. It follows a normal probability distribution $\mathrm{N}\left(0, \sigma_{\mathrm{E}}\right)$ of mean 0 and $\mathrm{SD} \sigma_{\mathrm{E}}\left(\sigma_{E}=\right.$ $0.075) . e_{L M}$ is drawn for every arbor and accounts for the variability of individual arbors' initial position. It follows a normal distribution $\mathrm{N}(0$, $\left.\sigma_{\mathrm{e}}\right)\left(\sigma_{\mathrm{e}}=0.025\right)$. For each potential arbor of an RGC located at position $x_{N T}$ along the N-T axis, we draw $y_{A P}$ randomly, with a uniform probability along the A-P axis. Then, the $y_{A P}$ position is retained with probability $P_{A}\left(x_{N T}, y_{A P}\right)$. When necessary, $y_{A P}$ is redrawn until a position is retained. Once all potential arbor positions have been calculated, we keep $n_{a}$ of them for each RGC. The selected arbors are the ones with the highest $P_{A}\left(x_{N T}, y_{A P}\right) \times N_{s}$ scores, where $N_{s}=\mathrm{N}\left(1, \sigma_{N_{s}}\right)$ is a multiplicative noise drawn for each arbor $\left(\sigma_{\mathrm{Ns}}=0.015\right)$, which models the imprecision of molecular signaling.

$P_{F}$ and $P_{R}$ are modeled as two-variable sigmoidal functions (see explanation in the Results section). Considering the orientation of molecular gradients in the retina and SC, $P_{F}(x, y)$ increases, and $P_{R}(x, y)$, decreases as $x$ gets more nasal or $y$ gets more anterior. Specifically, we choose $P_{F}$ and $P_{R}$ of the form:

$$
P_{Z}(x, y)=S_{Z}\left(B_{Z}(x, y)\right)=1 /\left(1+\exp \left(-r_{Z} B_{Z}(x, y)\right)\right), \quad Z=F \text { or } R,
$$

where $r_{Z}$ defines the slope of the sigmoidal envelope $S_{Z}(b)=1 /(1+\exp (-$ $\left.\left.r_{Z} b\right)\right)\left(r_{F}>0\right.$ and $\left.r_{R}<0\right)$, and $B_{Z}$ defines the shape of the map. In our simulations, we generally use $B_{Z}(x, y)=\left(b_{Z}^{T}-b_{Z}^{N}\right) x+b_{Z}^{N}-y$, where $b_{Z}^{N}$ and $b_{Z}^{T}$ parameterize $B_{Z}$ and are expressed as percentages along the A-P axis ( $b_{Z}^{N}$ and $b_{Z}^{T}$ can be negative or $>100 \%$ ) (Table 1$)$. The level set $P_{Z}=1 / 2$ or, equivalently, $B_{Z}=0$; hence follows the equation:

$$
y=\left(b_{Z}^{T}-b_{Z}^{N}\right) x+b_{Z}^{N},
$$

a line running between the points of coordinates $\left(0 \%, b_{Z}^{N}\right)$ and $\left(100 \%, b_{Z}^{T}\right)$ in the N-T/A-P axes. In EphA3 knock-in mice simulations, we choose an exponential $B_{Z}$ to simulate a saturation of ephrin-A inhibitory response to additional EphA3 (see explanation in the Results section). The exponential level sets $P_{Z}=1 / 2$ have the form:

$$
y=b_{Z}^{N}+\left(b_{Z}^{N}-b_{Z}^{T}\right)\left(e^{-c_{Z} x}-1\right) /\left(1-e^{-c_{Z}}\right),
$$

so that $y(0 \%)=b_{Z}^{N}, y(100 \%)=b_{Z}^{T}$, and $y^{\prime}(0 \%)=c_{Z}\left(b_{Z}^{N}-b_{Z}^{T}\right) /$ $\left(e^{-c_{Z}}-1\right) \cdot c_{Z}$ is a parameter controlling the convexity of the exponential boundary (Table 1), whose curvature increases as $c_{Z}$ increases. This formulation includes the linear case, corresponding to a "flat" exponential obtained when $c_{Z} \rightarrow 0$. We specifically parameterized Equation 3 to display this property. Exponential functions are not derived from any assumption of our model but are simply chosen for their convex shape and their simplicity.

Dynamic phase. The initial arborization is then fed to the dynamic phase, which is instructed by a combination of Hebbian arbor plasticity induced by retinal waves and competition for collicular resources, modeled by a single differential equation composed of two terms (Eq. 4). The derivation of the first term of the equation is provided in the next section:

$$
\begin{aligned}
\frac{d C(x, k)}{d t}=\alpha \sum_{\left(x^{\prime}, i\right)} W\left(x^{\prime}-x\right) & F\left(C\left(x^{\prime}, i\right)-C(x, k)\right) \\
& -\beta \nabla D_{C}(C(x, k)), \quad \forall(x, k),
\end{aligned}
$$

where $x$ is the discrete 2D position of an RGC in the retina, and $C(x, k)$ is the $2 \mathrm{D}$ collicular position of its $k$ th arbor, noted $(x, k)$. Arbor $(x, k)$ is subject to both Hebbian plasticity driven by retinal waves ("Hebbian term," sum weighted by $\alpha$ ) and axonal competition ("competition term," gradient weighted by $\beta$ ), which result in the evolution of its position $C(x, k)$. See Table 1 for the choice of $\alpha$ and $\beta$. When cell $x$ is active, it is so together with other cells $x^{\prime}$, which participate in the same waves and are correlated with $x$, on average, with correlation $W\left(x^{\prime}-x\right)$. We choose $W$ to be a Gaussian function centered in 0 , of SD $\rho$ (Table 1), only depending on the distance $\left\|x^{\prime}-x\right\|$ between $x$ and $x^{\prime}$. This choice is motivated by the experimental quantification of $W$ in Stafford et al. (2009). $W$ encodes in a very compact format the correlative effects of retinal waves; thus, we need not model individual retinal waves. Nonlinear function $F$ models the "Hebbian attraction" that arbor $\left(x^{\prime}, i\right)$ exerts on arbor $(x, k)$, and depends on the distance between them. We choose:

$$
F(y)=\left\{\begin{array}{ll}
K\left(1-\frac{\|y\|^{2}}{\theta^{2}}\right) \frac{y}{\theta}, & \text { if }\|y\| \leq \theta \\
0 & \text { otherwise }
\end{array} .\right.
$$

$\|F\|$ peaks at $\|y\|=\theta / \sqrt{ } 3$, with a value of 1 if we choose a normalization factor $K=3 \sqrt{3} / 2$. Our particular choice of function $F$ is justified in the next section.

As for the competition term, if $y$ is a $2 \mathrm{D}$ position in the SC, $D_{C}(y)$ describes the local density of RGC axonal processes, or equivalently the local lack of resources:

$$
D_{C}(y)=\sum_{(x, k)} G\left(C(x, k), \sigma_{a}\right)(y),
$$

the sum of small 2D Gaussian patches $G\left(C(x, k), \sigma_{a}\right)$ centered at arbor positions $C(x, k)$, with $S D=\sigma_{a}$, modeling spatially extended arbors ( $\sigma_{a}=6 \%$ of SC size). $\nabla D_{C}$ is the $2 \mathrm{D}$ gradient of this density so that $-\nabla D_{C}(C(x, k))$ is a diffusion term modeling how arbors tend to move down the local axonal density gradient. Our competition model is hence largely phenomenological and does not include assumptions about the possible mechanisms enforcing resource optimization.

Derivation of the Hebbian term. As retinal cell $x$ fires, the instant correlated activity at the level of RGCs' axonal arbors in the SC is, on average:

$$
I_{x}(y)=\sum_{\left(x^{\prime}, i\right)} W\left(x^{\prime}-x\right) f_{I}\left(y-C\left(x^{\prime}, i\right)\right),
$$


where $y$ is a 2D position in the SC and $f_{I}$ a decreasing, circularly symmetric function (i.e., depending only on the distance between its center and its argument) centered in 0, modeling spatially extended arbors in the SC. Possible choices for $f_{I}$ include a $2 \mathrm{D}$ Gaussian with $\mathrm{SD}=\sigma_{a}$ or a constant function defined on a disk of radius $\sigma_{\mathrm{a}}$, where the previously introduced quantity $\sigma_{\mathrm{a}}$ approximates the half-diameter of an arbor in the SC.

We hypothesize that active axonal processes activate the collicular dendrites they contact and that dendritic activity is integrated linearly at the level of collicular cell bodies. We introduce $f_{D}$, a decreasing, circularly symmetric function of radius $\sigma_{\mathrm{d}}$, representing spatially extended collicular dendritic trees. The distributed activity of collicular cell bodies can thus be written as a convolution of $I_{x}$, the net instant output of axonal arbors, with $f_{D}$. We then assume that stimulated collicular cells send a retrograde plasticity signal $R_{x}$ through their whole dendritic trees, promoting local arbor elaboration or stabilization. $R_{x}$ is then naturally modeled as a convolution of the activity of collicular cell bodies with the operator representing dendrites, $f_{D}$. In summary, $R_{x}$ is obtained by a double convolution of $I_{x}$ with $f_{D}$ :

$$
R_{x}(y)=f_{D} \otimes f_{D} \otimes I_{x}=\sum_{\left(x^{\prime}, i\right)} W\left(x^{\prime}-x\right) f_{R}\left(y-C\left(x^{\prime}, i\right)\right),
$$

where $f_{R}=f_{D} \otimes f_{D} \otimes f_{I}$ and $\otimes$ denotes the convolution operator. Because of the mathematical properties of convolution, $f_{R}$ is a decreasing, circularly symmetric function with a radial extent of the order of $\sqrt{\sigma_{\alpha}^{2}+2 \sigma_{d}^{2}}$.

Following the temporal correlation principle of Hebbian plasticity, the retrograde signal at the level of active collicular neurons' dendrites, $R_{x}$, affects arbors $(x, k), 1 \leq k \leq n_{a}$, which are simultaneously active. The retrograde signal in the vicinity of arbor $(x, k), R_{(x, k)}$, is a truncated version of $R_{x}$ :

$$
R_{(x, k)}(y)=M(y-C(x, k)) R_{x}(y),
$$

where $M(y-C(x, k))$ is a circularly symmetric mask, which is nonzero only in the vicinity of $(x, k)$ and has a radial extent of the order of $\sigma_{a} . M$ hence models the area over which the axonal arbor physically integrates retrograde signals, and $R_{(x, k)}$ is the part of the retrograde signal that is actually "seen" by the axonal processes of arbor $(x, k)$.

We assume that plasticity mediated by retrograde signals causes modifications of axonal processes that will tend to elaborate in regions with more retrograde signal and retract from areas with low signal, resulting in shifts of arbor centers. The following derivations then largely result from this additional "layer" of modeling and should not be seen as a mere derivation of the previous equations. We reduce the distribution $R_{(x, k)}$ (y) to a single center of mass:

$$
\frac{\int y R_{(x, k)}(y) d y}{\int R_{(x, k)}(y) d y}
$$

which defines the direction along which $(x, k)$ moves as its processes are reorganized through retrograde signaling. The axonal arbor does not have to "know" where the center of mass of the retrograde signal is since this information is naturally integrated in the modifications of its processes. The amplitude of the shift is not encoded in the center of mass and arguably depends on the intensity of the retrograde signal. For simplicity, we assume that the amplitude of the shift is proportional to the total retrograde signal integrated by the arbor. So, the spatial shift of arbor $(x, k)$ due to Hebbian plasticity, $\Delta C(x, k)$, is given by the following:

$$
\Delta C(x, k) \propto \int R_{(x, k)}(y) d y \times\left(\frac{\int y R_{(x, k)}(y) d y}{\int R_{(x, k)}(y) d y}-C(x, k)\right) .
$$

The symbol " $\propto$ ” means "proportional to." Developing Equation 11, we get:

$$
\Delta C(x, k) \propto \int R_{(x, k)}(y)(y-C(x, k)) d y .
$$

Replacing $R_{(x, k)}$ by the expressions in Equations 8 and 9, and developing the equation, we obtain:

$$
\begin{array}{r}
\Delta C(x, k) \propto \sum_{\left(x^{\prime}, i\right)} W\left(x^{\prime}-x\right) \int f_{R}\left(y-C\left(x^{\prime}, i\right)\right) \\
M(y-C(x, k))(y-C(x, k)) d y .
\end{array}
$$

We now define:

$$
F(z)=\int f_{R}(y-z) M(y) y d y
$$

If small border effects are neglected, a simple change of variable shows that:

$$
\begin{aligned}
F\left(C\left(x^{\prime}, i\right)-C(x, k)\right) & =\int f_{R}\left(y-C\left(x^{\prime}, i\right)\right) \\
& M(y-C(x, k))(y-C(x, k)) d y .
\end{aligned}
$$

Using Equation 15 in Equation 13, we obtain:

$$
\Delta C(x, k) \propto \sum_{\left(x^{\prime}, i\right)} W\left(x^{\prime}-x\right) F\left(C\left(x^{\prime}, i\right)-C(x, k)\right),
$$

and end up with the Hebbian term of Equation 4.

From its definition in Equation 14, we can derive several constraints that $F$ has to verify. First, as an integral, $F$ is continuous. In addition, one can easily prove, based on the properties of $f_{R}$ and $M$, that $F$ is circularly symmetric, centered in 0 , and that vector $F(z)$ points in the direction of $z$. Moreover, $\|F(z)\|$ is zero when $z=0$, and tends toward 0 when $\|z\|$ increases and is large enough. At this point, we could have calculated $F$ by choosing specific functions $f_{I}, f_{D}$, and $M\left(f_{R}=f_{D} \otimes f_{D} \otimes f_{I}\right)$ and using Equation 14, which would have led to intractable formulas. However, our derivation of $F$ so far did not require us to specify $f_{I}, f_{D}$, and $M$ and we used this freedom to design $F$ at our convenience. In this study, we opt for $F$ as in Equation 5, which has several advantages: it is very simple, allows rapid computations, and complies with all the above constraints. To our knowledge, the shape of function $F$ derived from our approach is novel and fundamentally different from functions used in previous similar models.

Simulation of the dynamic phase. Equation 4 was simulated with MATLAB (The MathWorks) using the Euler method as the integration scheme. The Hebbian term was normalized by the number of RGCs $(10,000)$ to render its amplitude independent from the chosen number. Density $D_{C}$ was sampled on a $512 \times 512$ discretized version of the SC. It was calculated as a convolution or, equivalently, as a product in the Fourier space of the distribution of arbor centers and a Gaussian patch representing their spatial extent $\left(S D=\sigma_{a}\right)$. It was then normalized by a constant normalization matrix that compensated for border effects (i.e., lower estimated density at the borders of the SC) and guaranteed that the amplitude of the competition term is independent of changes in $n_{a}, N_{r}$, or $\sigma_{a}$. Typical simulations had $T=50$ time steps and required $\sim 5$ min of computation time on a $2.3 \mathrm{GHz}$ Intel Core i5 MacBook Pro (Apple) with $4 \mathrm{~GB}$ of memory.

Simulation of phenotypes and quantification. To simulate retinotopic maps of individual visual axes, as obtained by intrinsic imaging (Kalatsky and Stryker, 2003; Cang et al., 2008), we plotted arbor centers as dots in the $2 \mathrm{D}$ continuous SC $\left(30,000\right.$ dots, $n_{a}=3$ arbors for each of the $N_{r} \times N_{r}=10,000$ RGCs) and color coded them according to their retinal origin along either the azimuth or the elevation visual axis. The fraction of the area of the SC covered by arbors was evaluated by computing the density $D_{C}$ with $\sigma_{a}=0.5 \%$, on a $512 \times 512$ grid and thresholding it so that density values below the 
Table 1. Simulation parameters

\begin{tabular}{|c|c|c|c|c|c|c|c|c|c|c|c|}
\hline Genotype & $b_{F}^{N}$ & $b_{F}^{T}$ & $b_{R}^{N}$ & $b_{R}^{T}$ & $c_{F}$ & $C_{R}$ & $T$ & $\alpha$ & $\beta$ & $\rho$ & $\theta$ \\
\hline WT & 140 & 35 & 45 & -15 & 0 & 0 & 50 & 0.6 & 1 & 0.05 & 0.25 \\
\hline ephrin-A-TKO & $\mathrm{N} / \mathrm{A}$ & $\mathrm{N} / \mathrm{A}$ & $\mathrm{N} / \mathrm{A}$ & $\mathrm{N} / \mathrm{A}$ & 0 & 0 & 100 & 0.6 & 1 & 0.05 & 0.25 \\
\hline EphA3-KI/KI & $140 / 30$ & $40 / 15$ & 40 & -15 & $0 / 4$ & 0 & 50 & 0.6 & 1 & 0.06 & 0.2 \\
\hline EphA3-KI/+ & $140 / 85$ & $30 / 25$ & 50 & -15 & $0 / 5$ & 0 & 50 & 0.5 & 1 & 0.05 & 0.2 \\
\hline EphA7-K0 & 140 & 35 & $\mathrm{~N} / \mathrm{A}$ & $\mathrm{N} / \mathrm{A}$ & 0 & 0 & 50 & 0.6 & 1 & 0.06 & 0.3 \\
\hline $\mathrm{p} 75^{\mathrm{NTR}}-\mathrm{KO}$ & 140 & 35 & 45 & -15 & 0 & 0 & 50 & 0.6 & 0.7 & 0.05 & 0.25 \\
\hline
\end{tabular}

threshold (0.05) were set to 0 and values above the threshold were set to 1 . This resulted in a $512 \times 512$ matrix $\left(a_{i j}\right)_{i, j}$ composed of zeros and ones. The fraction was then obtained as $\Sigma_{i, j} a_{i j} / 512^{2}$.

Anterograde tracings, as obtained by focal DiI injections in the retina (Feldheim et al., 1998; Frisén et al., 1998; Brown et al., 2000; Rashid et al., 2005; Pfeiffenberger et al., 2006; Lim et al., 2008), were simulated from groups of $10 \times 10$ RGCs ( $1 \%$ of the retina) representing focal injection loci. We modeled their arbors as small Gaussian patches $(S D=2.5 \%$ of SC size) and added them to obtain their axonal projections in the SC. Tracings were usually made from the $\mathrm{D}-\mathrm{V}$ center of the retina, in nasal (within 0-30\%), central (35-65\%), and temporal (70-100\%) locations along the N-T axis. The size of projection patches from anterograde tracings was evaluated as the $S D$ of the distribution of arbor centers in the SC and was expressed as a percentage of the length of the SC.

We quantified local map smoothness as a ratio $s(y)=r_{0} / r(y)$. Given a location $y$ in the SC, we considered that every arbor close enough to $y$ $(<5 \%$ of the SC size) was "seen" by a hypothetical collicular neuron in $y$, thanks to the extent of retinal axons and collicular dendrites. $r(y)$ is the spatial scatter of the distribution of RGCs corresponding to these arbors, and $r_{0}$ is a normalization constant (the value of the scatter in a flawless retinotopic map).

Parameters. Table 1 contains the parameters we used to simulate the arborization and dynamic phases in various scenarios. $b_{F}^{T}, b_{F}^{N}, b_{R}^{T}, b_{R}^{N}, c_{F}$, and $c_{R}$ describes the arborization phase. $T$ (number of integration steps), $\alpha, \beta, \rho$, and $\theta$ describe the dynamic phase. Other basic parameters were unchanged: $N_{r}=100, d t=1$ (integration step for the Euler method), $n_{p}=7, n_{a}=3, \sigma_{E}=0.075, \sigma_{e}=0.025, \sigma_{N s}=0.015$, and $\sigma_{a}=6 \% . r_{F}=20$ and $r_{R}=20$, except for the p $75^{\text {NTR }}$-KO case, where we used a modified sigmoid to account for altered reverse signaling: $S_{R}$ was linearly "compressed," to range between 0.96 and 1 instead of 0 and 1 , and the slope was set to $r_{R}=10$.

\section{Results}

In this study, we take a parsimonious approach and model development at the system's level in an effort to clarify how the complex interplay between molecular signaling, plasticity, and competition produces mature retinotopic maps. In our framework, the retina is simulated as a discrete $N_{r} \times N_{r}$ array of RGCs $\left(N_{r}=100\right)$ and the SC as a square, continuous space. The model features three developmental mechanisms acting in two phases (Fig. 1).

The first phase, the arborization phase, models the initial branching and elaboration of RGC axons in the SC (Fig. 1A), roughly corresponding to postnatal day 0 (P0) to $\mathrm{P} 3-\mathrm{P} 4$ in mice (McLaughlin et al., 2003). RGC axons initially overshoot their termination zone (TZ) and preferentially arborize along certain portions of the collicular A-P axis (Yates et al., 2001; McLaughlin et al., 2003). These portions are largely defined by EphA/ ephrin-A signaling, but their specific defining rules still lack supporting experimental evidence (McLaughlin and O'Leary, 2005; Dhande et al., 2011). In our model, each RGC establishes $n_{a}$ elementary axonal arbors $\left(n_{a}=3\right)$, and we simply assume that these arbors selectively develop in regions of the SC in which inhibition from forward and reverse molecular signaling is lower (Fig. 1A, colored areas in the SC).
The outcome of the initial phase is fed to the dynamic phase, which models the remodeling of the retinocollicular map occurring from $\mathrm{P} 3-\mathrm{P} 4$ to $\mathrm{P} 8$-P9 in mice (McLaughlin et al., 2003). This phase is driven by a combination of activity-dependent arbor plasticity (Fig. 1B) and competition for collicular resources (Fig. $1 C)$. As retinal waves propagate across the retina, the axons of neighboring RGCs have correlated firing patterns (Fig. $1 B$, red RGCs and arbors) (Galli and Maffei, 1988; Meister et al., 1991; Butts and Rokhsar, 2001; Stafford et al., 2009), and regions of the SC that are innervated by these correlated afferents are in turn stimulated (Fig. $1 \mathrm{~B}$, orange area in the $\mathrm{SC}$ ). We assume that active RGC arbors preferentially elaborate in these simultaneously active regions, following a Hebbian scenario (see Materials and Methods) (Hebb, 1949). In our model, this translates into shifts of active arbors toward regions with high collicular activity, that is, high concentrations of active arbors (Fig. $1 \mathrm{~B}$, blue arrows). Arbors from neighboring RGCs thus tend to converge toward each other if they are close enough in the SC. We term this effect Hebbian attraction. In addition, we assume that retinal axonal arbors compete for local collicular resources such as neurotrophins or collicular dendritic space (see Materials and Methods) (Cohen-Cory and Fraser, 1995; Ruthazer and Cline, 2004). In our model, arbors thus tend to develop away from areas with high arbor density (Fig. 1C, green area). This is modeled by shifts of arbor positions down the local gradient of arbor density (Fig. 1C, blue arrows).

Although the developmental factors considered in our model could partially overlap, we simulate retinocollicular development in two consecutive stages: initial arborization and maturation of the map. Doing so we do not advocate for an abrupt separation between the two phases or the corresponding mechanisms during development. However, several studies suggest that crude retinotopic maps that are formed by P3-P4 in mice could principally depend on molecular signaling, while the subsequent refinement mainly relies on Hebbian plasticity driven by retinal waves (Goodman and Shatz, 1993; Ruthazer and Cline, 2004; Pfeiffenberger et al., 2006; Cang et al., 2008). Such a scenario also received computational support from the model presented in Godfrey et al. (2009), in which the authors showed that the premature involvement of activity-dependent refinement, overlapping with the action of molecular cues, perturbs the development of retinotopic maps. Our choice of two consecutive phases, grounded in modeling parsimony and computational simplicity, is thus a reasonable approximation supported by both experimental and computational evidence.

Throughout this study, the complex axonal arbors of individual RGCs are represented by a greatly simplified ensemble of $n_{a}$ elementary arbor centers. We do not mean that each RGC has $n_{a}$ independent punctum-like arbors. Rather, arbor centers loosely describe possible underlying distributions of RGC axonal processes, as illustrated in Figure $1 D, E$. An RGC may have dense elaborations when elementary arbors coincide (Fig. $1 D$ ) or sparse 


\section{A}

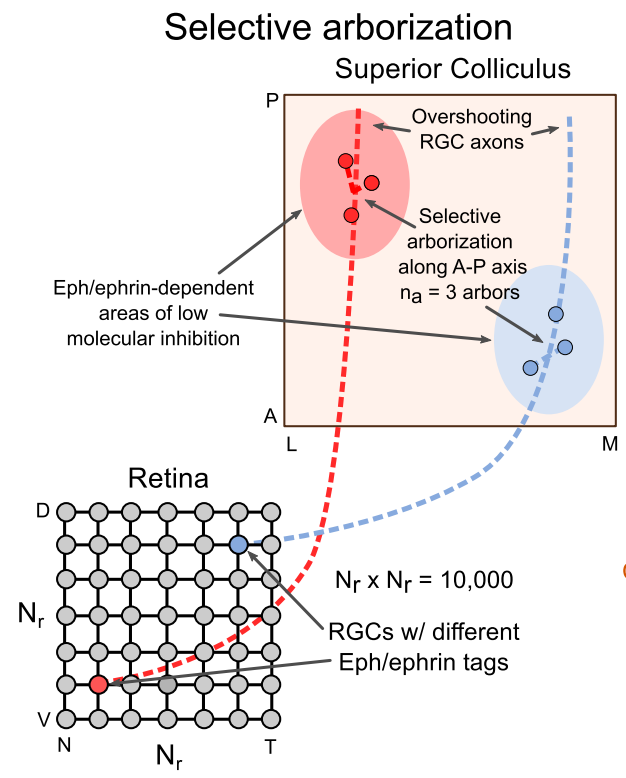

B
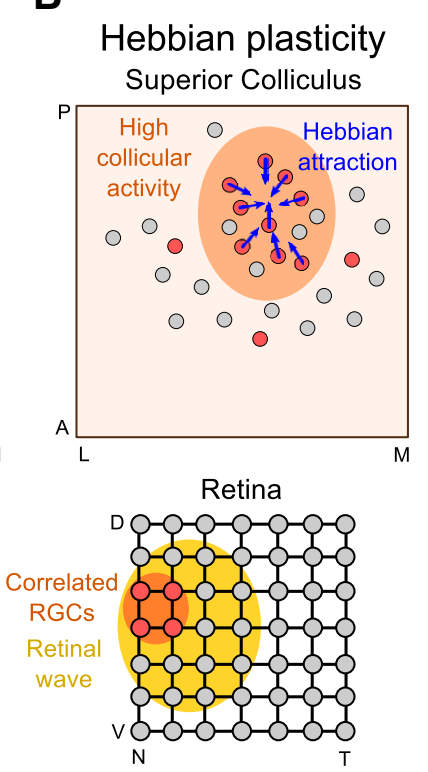

C

\section{Competition for resource}

Superior Colliculus

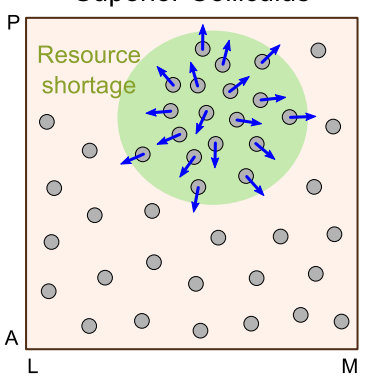

Retina

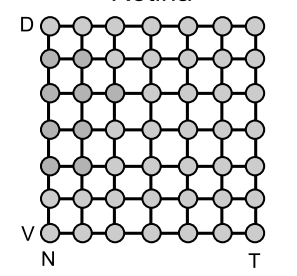

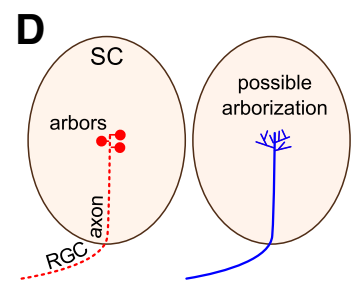
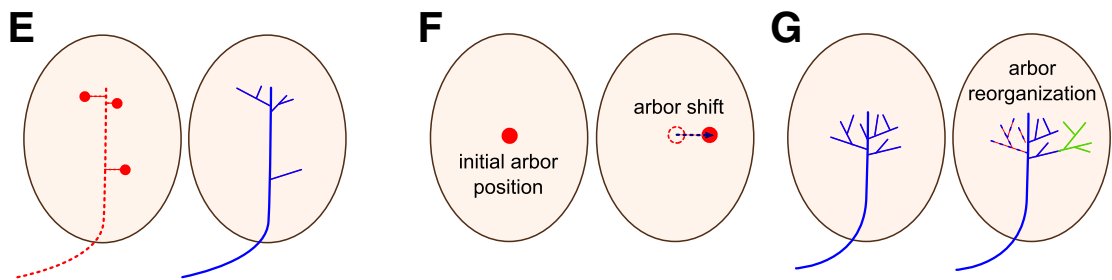

Figure 1. General principles of the model. $A-C$, The model features $N_{r} \times N_{r}=10,000 \mathrm{RGCs}$ arranged on a square grid, each projecting $n_{a}=3$ axonal arbors in a $2 \mathrm{D}$ continuous $S C$. $A$, Selective arborization phase. Molecular signaling mediated by Eph/ephrin gradients defines areas of low molecular inhibition along RGC overshot axons spanning the A-P axis of the SC (colored areas in the $\mathrm{SC}$ ), where axons selectively arborize. $\boldsymbol{B}$, Hebbian plasticity. The activity of neighboring RGCs is correlated by retinal waves (red RGCs and red arbors in the SC) and triggers activity in the SC (orange area). A retrograde signal is sent by collicular neurons to simultaneously active arbors to promote arborization and stabilize existing arbors. This results in "Hebbian attraction" (dark blue arrows) between correlated arbors that are close in both their retinal origin and collicular position. C, Competition for resources. High axonal arbors' density in the SC leads to scarce resources (green area). Arbors compete for resources by "traveling" up the gradient of resources or, equivalently, down the gradient of axonal density (dark blue arrows). In our model, this process does not explicitly depend on molecular cue levels or the electrical activity of RGCS. $\boldsymbol{D}, \boldsymbol{E}$, Single-cell arborization based on the position of arbor centers. Left panels show the position of arbor centers (red dots) for a single RGC. Right panels illustrate a possible corresponding arborization (blue). $\boldsymbol{D}$, Example of dense arborization. $\boldsymbol{E}$, Example of isolated branch and mildly elaborated arbor. $\boldsymbol{F}, \boldsymbol{G}$, Illustration of axonal remodeling as an arbor shift. $\boldsymbol{F}$, Arbor shift. $\mathbf{G}$, Possible corresponding remodeling of arbor involving pruning of axonal processes (dashes) and growth of new branches (green).

branches when elementary arbors are isolated (Fig. $1 E$ ). We do not explicitly model axonal branches, terminals, or retinocollicular synapses, so that the state of the retinocollicular system is entirely contained in the positions of RGC elementary arbors. In this context, the effects of activity-dependent plasticity and competition are described by shifts of elementary arbors, which are a simple way of modeling the evolution of complex arbors by widespread expansion and pruning of branches (Fig. $1 F, G$ ).

\section{A new, permissive model of}

\section{EphA/ephrin-A-mediated arborization}

The arborization phase models the inhibitory effect of forward and reverse signaling, mediated by gradients of EphAs and ephrin-As, on the initial establishment of retinal axonal arbors in the SC. Our arborization model follows two steps. Each RGC first establishes a pool of $n_{p}=7$ potential axonal arbors in the SC and then selects and retains only the $n_{a}=3$ most "appropriate" arbors. Our two-step model including both arborization and pruning is supported by recent experimental evidence showing that between $\mathrm{P} 0$ and $\mathrm{P} 4$, axons of individual RGCs start by developing unelaborated tentative branches over a broad range of the SC's A-P axis and then form dense arbors in the vicinity of their correct TZ while eliminating inappropriate branches (Dhande et al., 2011). Both steps rely on a two-variable function $P_{A}$ quantifying the role of molecular signaling in mapping the $\mathrm{N}-\mathrm{T}$ axis of the retina onto the A-P axis of the SC. $P_{A}(x, y)$ depends on the position of an RGC along the retinal N-T axis $(x)$ and a potential position of one of its arbors along the A-P axis in the $\mathrm{SC}(y) . P_{A}(x$, y) $\epsilon[0,1]$ is the probability that this arbor develops at A-P position $y$, considering EphA and ephrin-A expression levels at positions $x$ and $y$ (Fig. 2A). $P_{A} \approx 0$ indicates strong inhibition, and $P_{A} \approx 1$ indicates permission to arborize. We assume that forward signaling (between collicular ephrin-As ligands and retinal EphA receptors) and reverse signaling (between collicular EphAs and retinal ephrin-As) are functionally independent. Their combined effect, quantified by $P_{A}$, is then the product of two independent functions, $P_{F}$ and $P_{R}$, modeling the respective inhibitory effects of forward and reverse signaling (Fig. $2 B, C$ ). There is currently no quantitative knowledge of how different concentrations of retinal and collicular EphA/ephrin-As mediate the inhibition of arborization, but a few qualitative rules have been experimentally established. First, gradients of EphA/ephrin-A expression along the retinal N-T axis or the collicular A-P axis are well approximated by an exponential plus a constant (Reber et al., 2004). Second, higher concentrations of retinal or collicular EphA/ ephrin-As lead to increased inhibition in both forward and reverse signaling (Flenniken et al., 1996; Rashid et al., 2005), and it is reasonable to assume that permission to arborize is inversely 
A

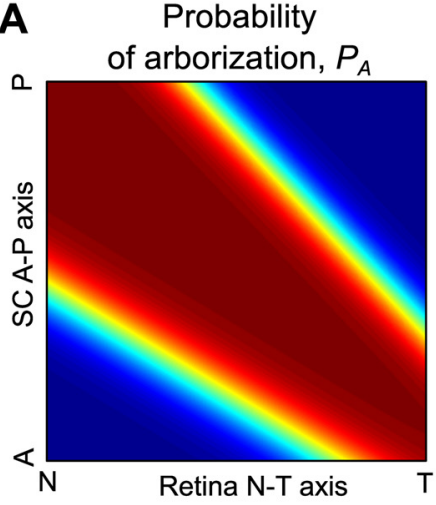

D Potential Arbors

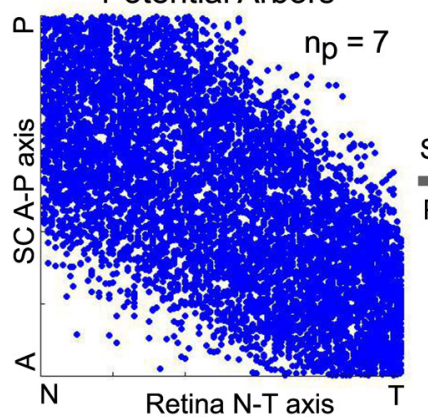

B
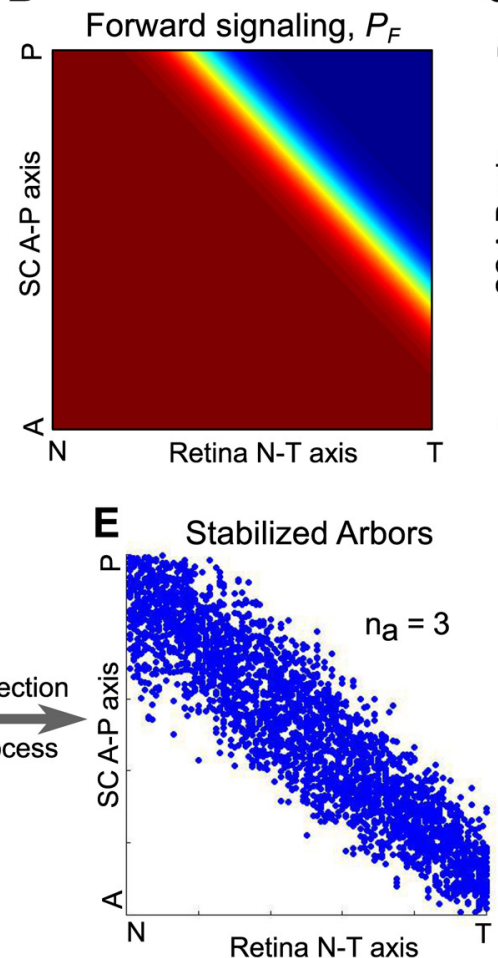

C

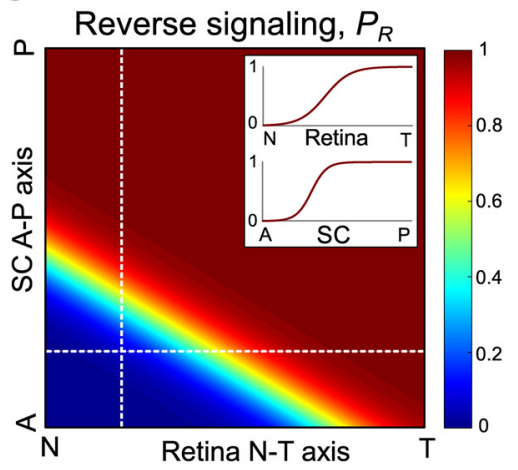

$\mathbf{F}$

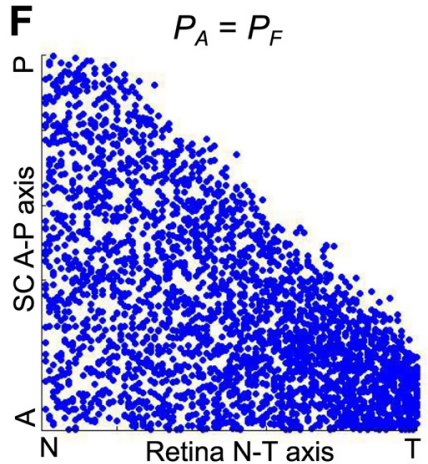

Figure 2. Arborization phase. $A-C$, Maps describing the inhibitory effect of EphA/ephrin-A signaling, depending on the N-T retinal position of an RGC and the A-P collicular position of its arbor. High collicular ephrin-A and retinal EphA (forward signaling) or high collicular EphA and retinal ephrin-A (reverse signaling) cause a decreased probability of arborization, modeling the repulsive interaction between EphA and ephrin-A. $P_{A}$ probability map for WT mice $(\boldsymbol{A})$, obtained by multiplying the maps of forward $(\boldsymbol{B})$ and reverse $(\boldsymbol{C})$ signaling. $\boldsymbol{C}$, Inset, Sigmoidal profiles of the map along the N-T and A-P axes, corresponding to the white dashed lines. $\boldsymbol{D}, \boldsymbol{E}$, Example of potential and stabilized initial arborization. N-T/A-P retinocollicular slices (representations in their N-T and A-P coordinates of all the arbor centers from a "slice" of the retina spanning the whole N-T axis and only of $10 \%$ of the D-V axis) showing arbor centers before $\left(\boldsymbol{D}, n_{p}=7 ; 7000\right.$ dots) and after $\left(\boldsymbol{E}, n_{a}=\right.$ 3; 3000 dots) selection of the best potential arbors. For each RGC, arbors with the highest $P_{A} \times N_{S}$ scores are retained. $\boldsymbol{F}$, Mapping obtained with forward signaling only $\left(P_{A}=P_{F}\right)$. Nasal axons do not make selective arborizations along the A-P axis, illustrating that our model is not based on gradient matching.

proportional to both receptor and ligand concentrations. As the inverse of an exponential plus a constant is a sigmoid, $P_{F}$ and $P_{R}$ should naturally have sigmoidal profiles along both the $\mathrm{N}-\mathrm{T}$ and A-P axes (Fig. 2C, dashed white lines and corresponding inset). The specific equations are listed in Materials and Methods.

We simulated the arborization phase in WT using the $P_{A}$ function in Figure $2 A$. The outcome of the first step is displayed in Figure $2 D$, in the form of an "N-T/A-P slice"-that is, a partial view of the mapping where only arbors arising from a subregion of the retina (here the central $10 \%$ of the retina along the $\mathrm{D}-\mathrm{V}$ axis, 7000 elementary arbors represented as blue dots) are displayed and projected onto the N-T and A-P axes. The slice shows potential arbors defining a very imprecise retinotopic mapping of the N-T axis onto the A-P axis. This reflects the broad potential of elaboration of bare axonal branches initially spanning large portions of the SC. At the second step, we assume that tentative axonal sprouts that are in an adverse molecular environment, corresponding to low $P_{A}$ values, do not develop, or eventually retract, as suggested experimentally (Dhande et al., 2011). Specifically, each RGC eliminates the arbors with the lowest $P_{A} \times N_{s}$ scores. Noise $N_{s}$ models the fact that gradients of molecular cues in the retina and SC are not perfectly regular and that inhibition mediated by molecular signals is probably not precise enough to discriminate potential arbors with almost identical $P_{A}$ scores. After the selection step, as in experimental data at $\mathrm{P} 4$, the retinotopic mapping is still immature and topographically imprecise but most of large potential errors have been avoided (Fig. 2E) (Dhande et al., 2011).
Importantly, our arborization process is not based on chemospecificity (i.e., gradient matching). Otherwise, forward signaling between retinal EphAs and collicular ephrin-As, both of which are expressed in gradients, could alone instruct the position of nasal axons. Instead, when $P_{A}=P_{F}$, nasal RGCs project everywhere along the A-P collicular axis because of their low levels of EphAs (Fig. 2F). Our model relies neither on instructive mechanisms such as axonal "repulsion" nor on "guidance," wherein axons move along molecular gradients until they encounter an appropriate "stop signal." The latter could be, as proposed in previous models, a match in retinal and collicular molecular cue levels, a balance between the opposing repulsive forces of forward and reverse signaling, or an equilibrium between gradient-based repulsion and other factors such as, for example, axonal competition (Simpson et al., 2009; Triplett et al., 2011). Instead, our arborization model features selective branching based on the total inhibition caused by independent forward and reverse signaling pathways. We term the model "permissive" because, contrary to previous models, it does not explicitly or implicitly rely on the assumption that EphA/ephrin-A signaling instructs axons' termination zones in the SC, even only coarsely.

The difference between our permissive model and previous models can be further illustrated in our simulations of EphA3 knock-in mice (Brown et al., 2000). EphA3 is normally absent from the mouse retinocollicular system. In these knock-in mice, EphA3 is expressed under the control of the Islet-2 gene (Isl2) and targets $\sim 50 \%$ of RGCs in a salt-and-pepper fashion across the retina. As a result, Isl2- RGCs express normal levels of EphAs, 
A

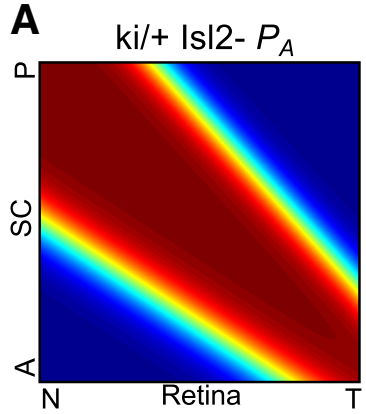

D $\quad k i / k i$ Isl2- $P_{A}$
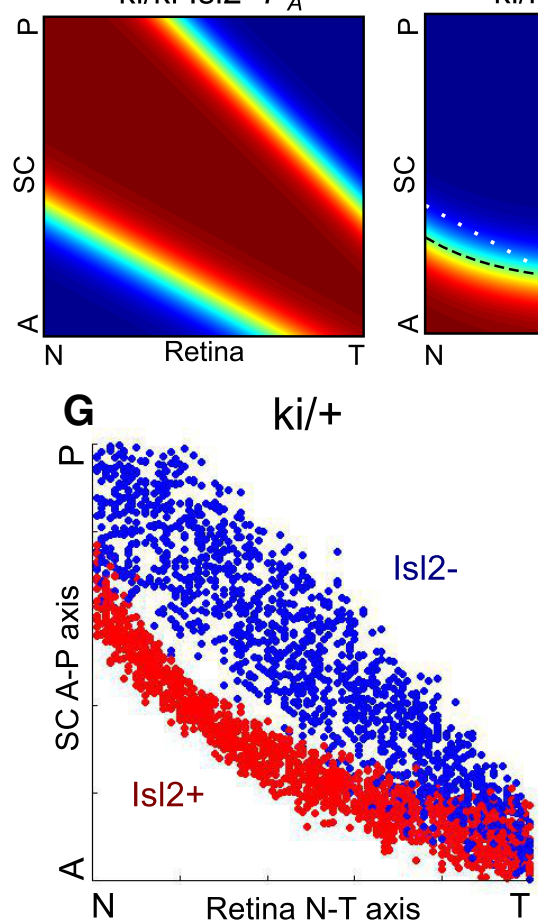

B $\quad \mathrm{ki} /+|\mathrm{s}| 2+P_{F}$

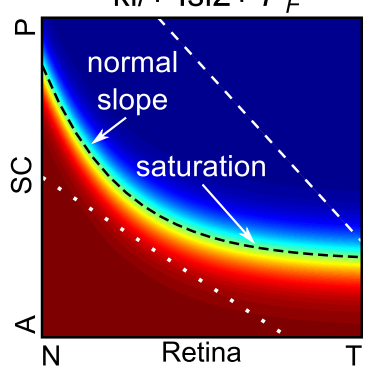

E

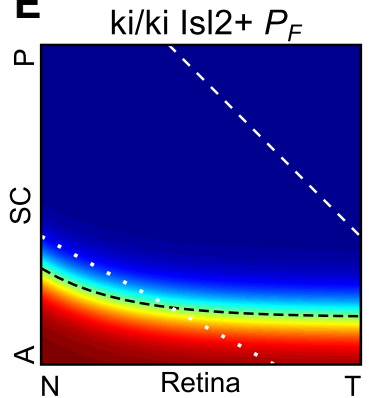

C
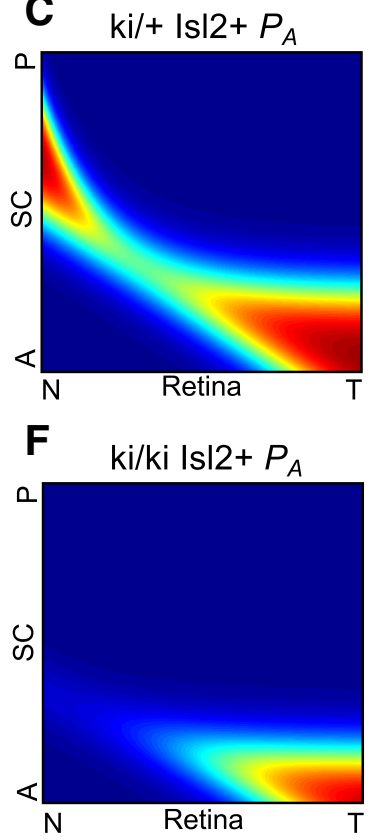

$\mathrm{ki} / \mathrm{ki}$

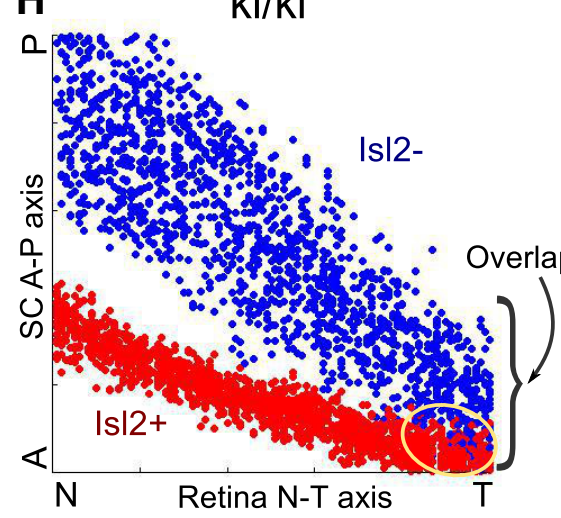

Figure 3. Arborization phase for EphA3-KI simulations. $\boldsymbol{A}-\boldsymbol{C}, \mathrm{ki} /+$ signaling. $\boldsymbol{A}, P_{A}$ for ki/ $+\mathrm{Is} \mid 2-\mathrm{RGCs} \operatorname{similar}$ to WT. $\boldsymbol{B}, P_{F}$ for $\mathrm{ki} /+|\mathrm{s}| 2-\mathrm{RGCs}$. The level set $P_{F}=1 / 2$ (black dashed line) shows the exponential profile of function $P_{F}$, modeling the saturation of the inhibitory effect of collicular ephrin-As on heightened retinal EphAs. The white dashed line indicates the same level set for the $|s| 2-P_{F}$ function, and the white dotted line indicates the curve $P_{R}=1 / 2 . \boldsymbol{C}, P_{A}$ for ki $/+|s| 2+R G C s . D-F$, ki/ki signaling. G, Arborization in the ki/+ case, displaying $|s| 2-$ (blue) and $|s| 2+\left(\right.$ red) arbors corresponding to the $P_{A} \sin \boldsymbol{A}$ and $\boldsymbol{C}$, respectively. $\boldsymbol{H}$, $\mathrm{ki} / \mathrm{ki}$ arborization using the $P_{A} s$ in $\boldsymbol{D}$ and $\boldsymbol{F}$. The coarse $|\mathrm{s}| 2-$ and $|\mathrm{s}| 2+$ mappings overlap over the anterior $40 \%$ of the $S C$ (accolade). Temporal Is|2- and $|s| 2+$ arbor populations are intermixed in the anterior SC (circled arbors).

goes beyond the WT maximum observed in temporal Isl2- axons. We model this effect by using $P_{F}$ maps with convex instead of linear shapes (see Materials and Methods) (Fig. 3B,E). For ki/+ nasal Isl2+ RGCs, which have EphA levels within the range observed in WT mice despite their additional EphA3 (Reber et al., 2004), the slope of $P_{F}$ was chosen similar to the Isl2- case (compare the nasal slope of the level set $P_{F}=0.5$ in Fig. $3 B$, black dashed line, with the slope of the same level set in the Isl2- case, white dashed line). On the other hand, the inhibitory effect on more temporal Isl2+ axons, whose EphA levels are beyond the WT range, was relatively diminished and saturated to reach a temporal boundary value closer to the one of Isl2 - temporal axons (compare temporal ordinates of black and white dashed lines) (Fig. 3B). Reverse signaling was the same in the Isl2- and Isl $2+$ case (Fig. $3 B, E$, white dotted lines). At the end of the arborization phase with such $P_{A}$ maps, the Isl2- axons (Fig. 3G, H, blue dots) behave largely like WT and occupy the entire collicular A-P axis, whereas Isl2 + axons are restricted to the anterior SC where collicular inhibition is lower (red dots). In the ki/ki case, Isl2- and Isl2 + arbor populations largely overlap in the anterior SC (Fig. $3 H$ ). In particular, despite their different levels of EphAs, Isl2and Isl $2+$ arbors from temporal RGCs project to the same anterior region in the SC, where inhibition is the lowest for both types of axons (circled arbors) (Fig. 3H). This outcome is fundamentally different from the models that assumed relative gradient matching, where RGC axons are "sorted" based on their relative EphA levels and consequently form a double map in the ki/ki case. In our permissive arborization model, the double map is not instructed by molecular signaling but appears in the dynamic phase as shown below. and Isl $2+$ cells express an increased level, including additional EphA3. Isl2 + RGCs express approximately twice as much additional EphA3 in the knock-in homozygotes (ki/ki) as in their heterozygote counterparts $(\mathrm{ki} /+)$. Consequently, $\mathrm{ki} / \mathrm{ki}$ mice show striking double retinotopic maps (Triplett et al., 2009), while ki/+ mice have two distinct maps that overlap along approximately $40-60 \%$ of the collicular A-P axis and "collapse" into a unique map in the anterior part of the SC (Brown et al., 2000). In our simulations, each RGC was randomly labeled "Isl2-" or "Isl2+" with a probability of 1/2. Isl2- RGCs had a normal $P_{A}$ (i.e., similar to the WT $P_{A}$ map used above) (Fig. $3 A, D$ ) and thus a normal arborization phase (Fig. $3 G, H$, blue dots). Isl2 + RGCs were subject to a more stringent forward signaling, modeling the effect of additional EphA3 (Fig. $3 B, C, E, F$ ). We further assumed that the global inhibitory response of heightened retinal EphAs to collicular ephrin-As saturates as EphA level
Balanced Hebbian plasticity and resource-based competition deeply remodel immature retinocollicular maps

We used a single differential equation to simulate Hebbian arbor plasticity and competition for collicular resources (Eq. 4; see Materials and Methods). In the WT case, the arborization phase generated a grainy retinotopic map (Fig. 4A, corresponding to Fig. $2 E$ ), with scattered anterograde projections (Fig. $4 B$ ) (see Materials and Methods). The map showed a global retinotopic organization, and projections were centered at roughly correct retinotopic locations, consistent with observations made in WT mice at approximately P4 (McLaughlin et al., 2003). This coarse retinotopic mapping was then fed into Equation 4. Over time, the size of anterograde projections decreased (Fig. $4 C$, solid blue curve) and the map became smoother (Fig. $4 D$, solid red curve). This resulted, at the end of the dynamic phase $(t=50)$, in a refined retinotopic mapping with a smooth map of the retinal 
A

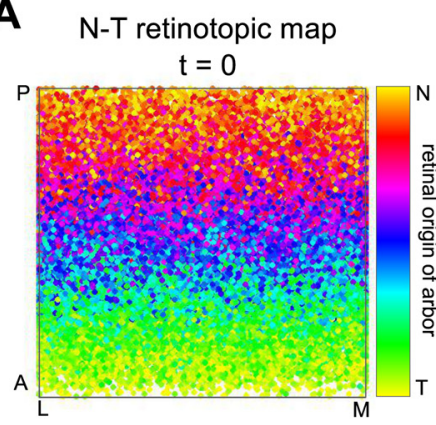

E
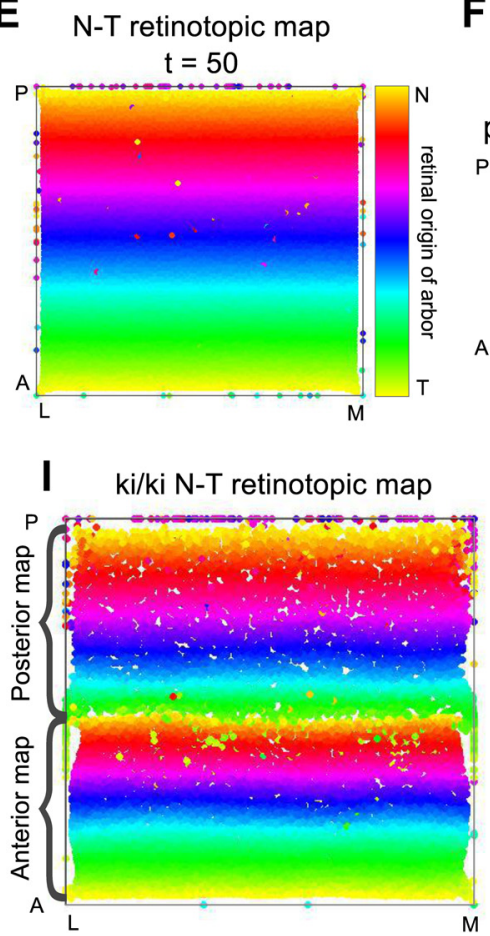

B

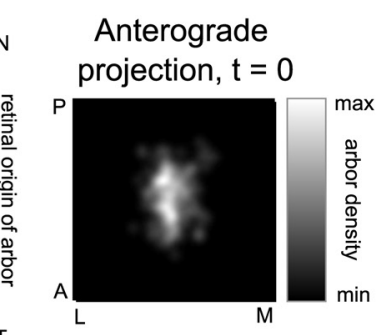

$\mathbf{F}$

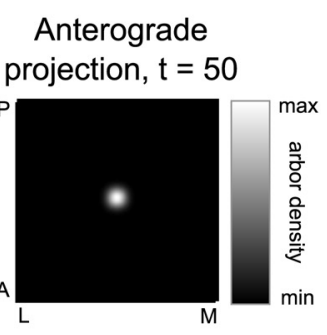

C Size of projection patches

$$
15 \text {. }
$$
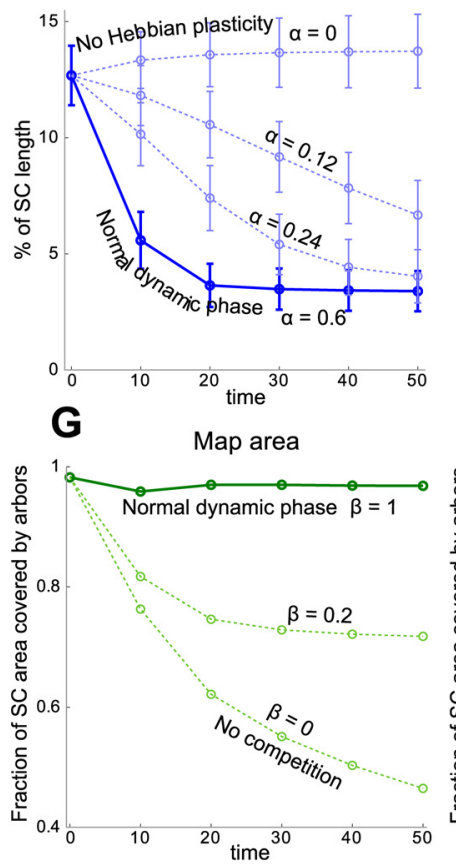

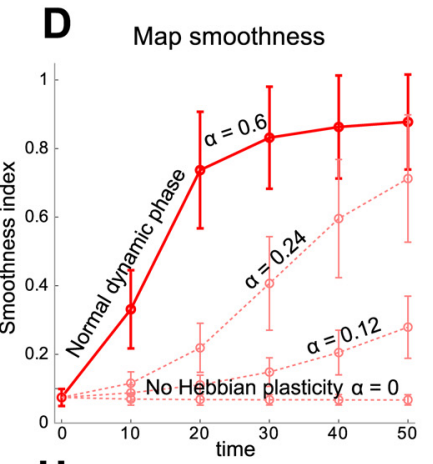

H

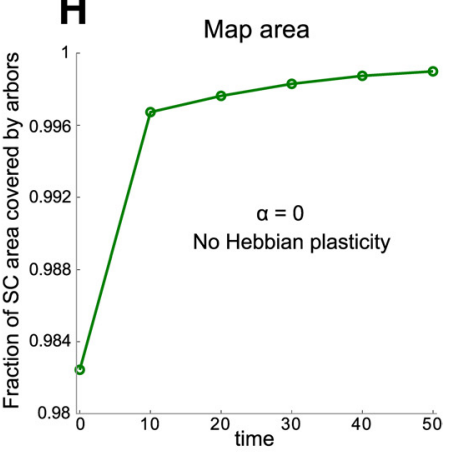

J $\quad \mathrm{ki} /+\mathrm{N}-\mathrm{T}$ retinotopic map

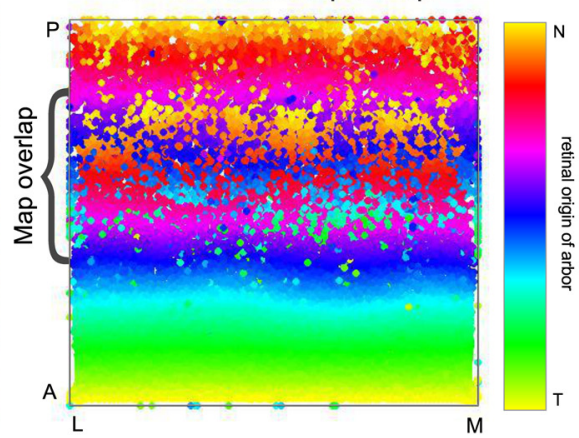

K ki/ki anterograde projections
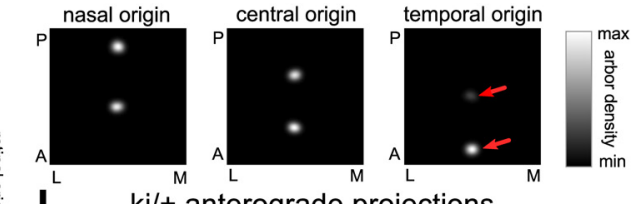

L $\quad \mathrm{ki} /+$ anterograde projections
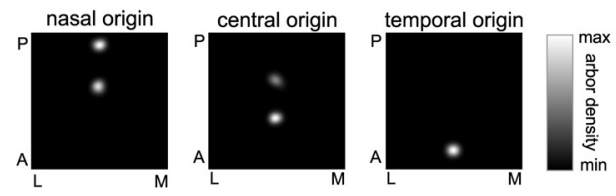

Figure 4. Dynamic phase. $\boldsymbol{A}, \boldsymbol{B}$, Retinocollicular phenotypes at the end of the arborization phase, consistent with observations in P4 mice. $\boldsymbol{A}$, Grainy retinotopic map, with an overall retinotopic structure. $\boldsymbol{B}$, Scattered anterograde tracing from the central retina, centered at a roughly correct retinotopic location in the SC. $\boldsymbol{C}, \boldsymbol{D}$, Time course over the dynamic phase of the size of anterograde projections $(\boldsymbol{C})$ and smoothness $(\boldsymbol{D})$, averaged from 100 tracing sites in the retina and 100 locations in the SC, respectively. The solid curve corresponds to the normal WT scenario, and dashed lines correspond to various smaller values of $\alpha$, for which projections are less refined $(\boldsymbol{C})$ and the map is less smoothed $(\boldsymbol{D})$. $\boldsymbol{E}, \boldsymbol{F}$, Retinocollicular phenotypes at the end of the dynamic phase, consistent with observations in P8 mice. $\boldsymbol{E}$, Smooth, mature retinotopic map. $\boldsymbol{F}$, Mature focal anterograde tracing from the center of the retina. $\boldsymbol{G}, \boldsymbol{H}$, Time course of the fraction of the collicular area occupied by all the arbors. G, A normal level of competition guarantees that the map does not shrink under Hebbian attraction (solid line). Lowered competition levels lead to shrunken maps (dashed lines). $\boldsymbol{H}$, Pure competition tends to stretch the map so that it fills an increasing area of the SC, though the effect is small in this example (note the $y$-axis scale). $\boldsymbol{I}-\boldsymbol{L}$, EphA3-KI simulations. $\boldsymbol{I}$, The ki/ki N-T retinotopic map has two submaps, indicated by accolades. J, The ki/ + map is normal in its anterior part ( $\sim 0-40 \%$ of the A-P axis), scrambled over approximately $40 \%$ of the central A-P axis (accolade) and normal over the most posterior $20 \%$. $\boldsymbol{K}$, ki/ki anterograde projections are double (temporal ones are indicated by red arrows). $\boldsymbol{L}$, ki/ + projections from the $20-25 \%$ most temporal part of the retina are single, and projections from the nasal and central retina are double.

$\mathrm{N}-\mathrm{T}$ axis onto the A-P axis of the SC as well as tight projections (Fig. $4 E, F$ ). These phenotypes are characteristic of $\mathrm{P} 8+\mathrm{WT}$ mice, in which the retinocollicular mapping is considered mature (Frisén et al., 1998; Cang et al., 2008). We studied the respective role of Hebbian plasticity and competition by modifying their contributions during the dynamic phase, starting from the same initial arborization. Hebbian plasticity is responsible for the refinement of the mapping, as increasing the corresponding parameter, $\alpha$, enhanced the refinement, and competition alone was ineffective (Fig. 4C,D, dashed curves). Reducing the contribution of competition, $\beta$, did not impair map refinement but resulted in shrunken maps, where arbors failed to occupy the whole area of the SC (Fig. $4 G$, dashed curves). In the absence of Hebbian plasticity, it appeared that competition "stretches" the map, so that arbors cover an increasing area in the SC (Fig. $4 H$ ).
We then used the projection patterns in Figure $3 G, H$ as initial conditions and simulated the dynamic phase in EphA3 knock-in mice. At the end of the dynamic phase, the ki/ki simulation displayed a double $\mathrm{N}-\mathrm{T}$ retinotopic map (Fig. $4 I$ and corresponding Fig. $5 B$ ). Naturally, anterograde tracings in $\mathrm{ki} / \mathrm{ki}$ mice were also double (Fig. $4 K$, some patches indicated by a red arrow). ki/ + simulations had a grainy $\mathrm{N}-\mathrm{T}$ retinotopic map around the middle of the A-P SC axis because, in this region, collicular neurons received two overlapping sets of projections from RGCs with distinct N-T retinal origins (Fig. $4 J$ and corresponding Fig. 5D). The most posterior and anterior parts of the map were smoother because the underlying projections had unique retinal origins. In ki/ + simulations, anterograde tracings from the $\sim 75 \%$ most nasal portion of the retina had two TZs, while tracings from the most temporal 

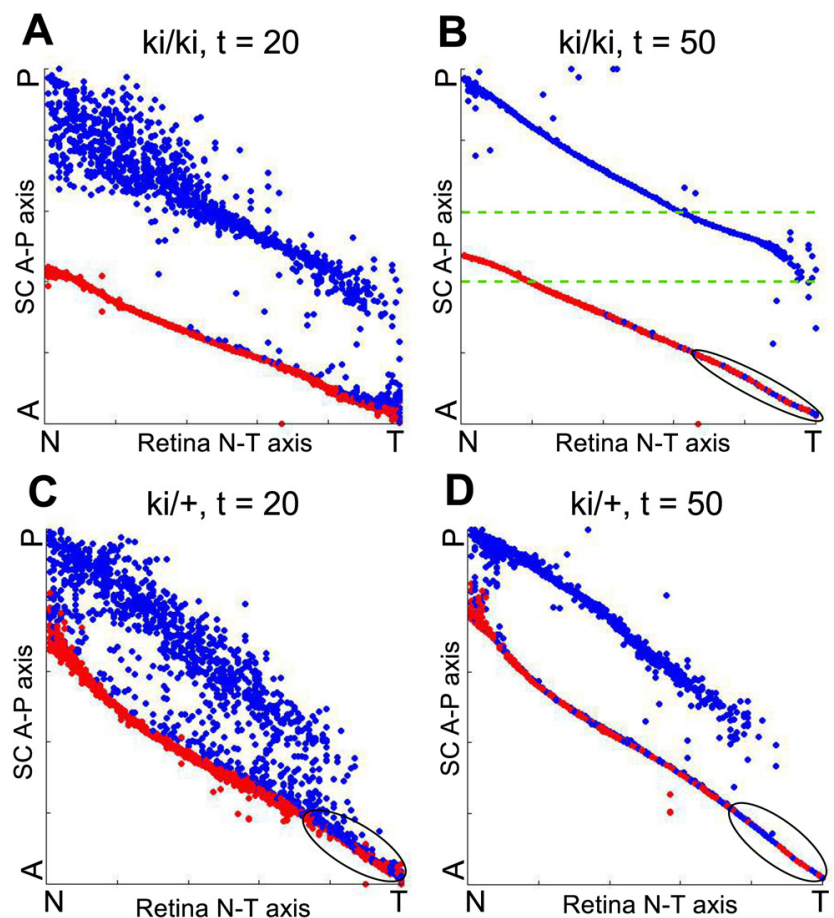

\section{E ki/ki: arbor count from central SC}

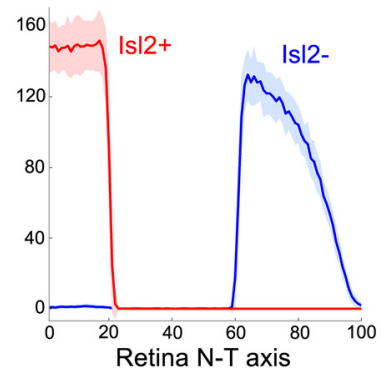

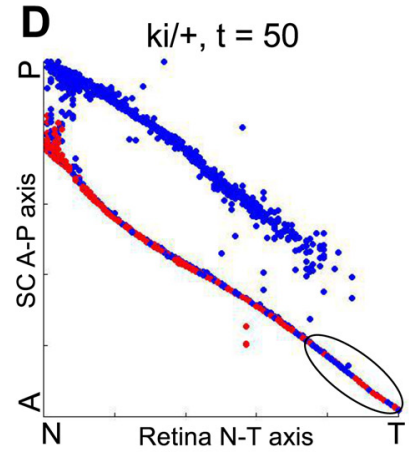

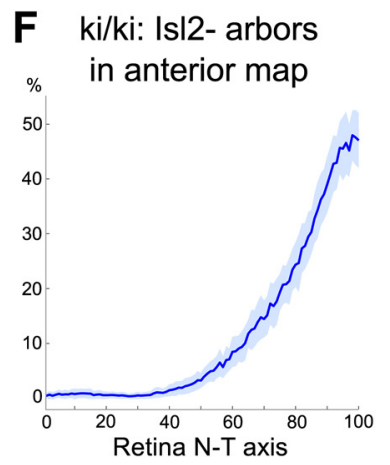

Figure 5. Repartition of $|s| 2+$ and $|s| 2-$ arbors in EphA3-KI simulations. $\boldsymbol{A}, \boldsymbol{B}$, Dynamic phase for the ki/ki arborization in Figure $3 H . A, A t t=20$, part of the temporal Is $2-$ arbors fuse with anterior $1 \mathrm{~s} \mid 2+$ arbors, and other temporal arbors elaborate more posteriorly, "pulled" by posterior $|s| 2-$ arbors, resulting in two separate layers. $\boldsymbol{B}$, Final double-map phenotype. The dashed green lines define a central A-P collicular domain (see $\boldsymbol{E}$ ). The anterior SC receives projection from both $|s| 2+$ and $I s \mid 2-$ axons (circled). C, D, Dynamic phase for the ki/ + arborization in Figure 3G. C, At $t=20$, temporal $|s| 2-$ and $|s| 2+$ arbors fuse into a single layer (circled arbors). D, Final "collapsed" map phenotype (circled). The anterior $40 \%$ part of the SC has a single map made of $|s| 2-$ and $|s| 2+$ arbors. The $40-80 \%$ portion of the mapping along the A-P axis has two overlapping maps. $\boldsymbol{E}$, Composition of axonal projection in the middle $20 \%$ of the SC delimited by the green dashed lines in $\boldsymbol{B}$ averaged over $50 \mathrm{ki} / \mathrm{ki}$ simulations (SD indicated in paler colors). These projections are almost exclusively made of nasal $|s| 2+$ and temporal $|s| 2-$ arbors. $\boldsymbol{F}$, Proportion of $|s| 2-$ arbors in the anterior map, averaged over 100 $\mathrm{ki} / \mathrm{ki}$ simulations, depending on the $\mathrm{N}-\mathrm{T}$ retinal position (standard deviation in pale blue). This curve shows a significant proportion of temporal Is 12 - arbors in the anterior layer of $\mathrm{ki} / \mathrm{ki}$ simulations.

part had only one TZ (Figs. $4 L, 5 D$ ), as observed experimentally (Brown et al., 2000).

How can we explain the appearance of these experimentally observed phenotypes, our ki/ + and ki/ki simulations? In projections from the nasal or central retina, Isl2 + and Isl2- arbors are initially well segregated along the A-P collicular axis, on the basis of EphA/ephrin-A signaling (Fig. 3G,H). Despite that this is not obvious in the figures, the second step of the arborization phase is essential to obtain this segregation, since it efficiently "cleans" each population from potential arbors of the other genotype.

This segregation is then conserved through the dynamic phase (Fig. 5A-D). However, projections from the temporal retina have intermixed Isl2 + and Isl2- arbors in the anterior part of the SC (Fig. 3G,H, circled arbors). In ki/ + simulations, Isl2+ arbors from temporal RGCs arborize in more anterior positions than their Isl2- counterparts, but the two populations of arbors largely mix with one another. During the dynamic phase, these two populations fuse into a single map because of Hebbian attraction, which leads to the experimentally reported "map collapse” (Fig. 5C,D, circled regions) (Brown et al., 2000). In ki/ki mice, the overlap between Isl2 + and Isl2 - arbors from temporal axons is less because Isl2 + axons arborize very anteriorly in the SC, due to their high EphA3 level (Fig. $3 H$ ). Then during the dynamic phase, arbors in the anterior SC aggregate with one of two separate "layers" of arbors, depending on their initial location along the A-P axis (Fig. $5 A, B$ ). More precisely, axons in the anterior SC rapidly form a dense layer, and temporal axons that have not arborized in the most anterior parts of the SC are subject to Hebbian attraction from more posterior arbors and intense competition from both nasal and temporal axons, which favors their elaboration in more posterior regions. Simulations of knock-in mice then illustrate how the topology of arbor distributions in the SC can be deeply modified by Hebbian plasticity and axonal competition to shape critical features that are not instructed by permissive molecular cues. Initially intermixed ensembles of arbors in the anterior colliculus are indeed segregated, and even torn apart, to form two separate maps (compare Figs. $3 H, 5 B)$.

Our successful simulation of EphA3 knock-in mice reveals an important difference between our model and other models regarding the nature of axonal competition. We have emphasized that axonal competition depending on available collicular resources is an important governing factor in retinotopic map formation, but other types of axonal competition have been proposed. A prominent theory posits that during retinocollicular development, RGC axons compete with each other on the basis of comparisons of their EphA receptor levels, resulting in the spatial segregation of retinal axons with different EphA levels (Brown et al., 2000; Reber et al., 2004). This theory predicts that in ki/ki mice, the posterior and anterior maps are entirely composed of Isl2 - and Isl2+ axons, respectively. Experimental tests in EphA3-KI homozygotes, where retrograde tracings from the middle of the SC back to the retina were performed, were consistent with that prediction (Brown et al., 2000). In these experiments, virtually all axons tracing back to the nasal retina, belonging to the anterior map, arose from Isl2+ cells, while axons tracing back to the temporal retina, belonging to the posterior map, came from Isl2- cells. This experimental result was reproduced in our simulations. We simulated retrograde tracings from the center of the SC by checking the origin of arbors present in the central $20 \%$ of the SC along the A-P axis (Fig. $5 B$, region between the green dashed lines). We indeed found that these arbors almost exclusively "traced back" to either nasal Isl2+ RGCs or Isl2 - temporal RGCs (Fig. 5E, averaged over 50 simulations). However, we also found that the anterior part of the anterior map (Fig. 5B, circled arbors) contained, besides temporal Isl2 + RGCs, a significant proportion of temporal Isl2- RGCs (Fig. 5F, averaged over 100 simulations). This result violates the principle of map separation between Isl2 - and Isl2 + axons suggested by the theory of EphA-based competition. Experimentally testing our prediction on RGC axons in the anterior SC could thus demonstrate that models of retinocollicular development 

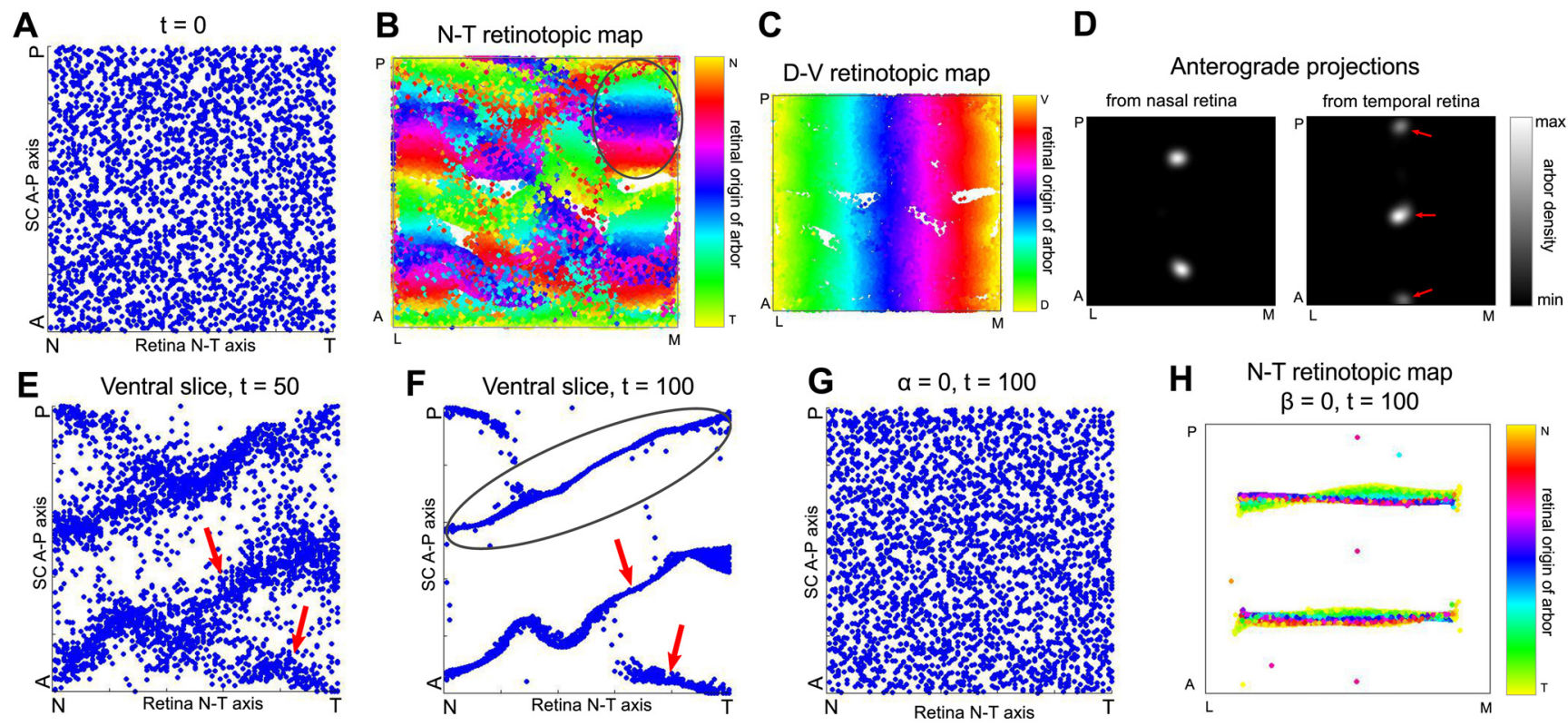

Figure 6. Retinocollicular mapping in the absence of molecular signaling. $\boldsymbol{A}-\boldsymbol{D}$, Simulation of retinocollicular mapping with random initial distribution of arbors along the A-P axis, reminiscent of ephrin-A TKO mice. $A, N-T / A-P$ slice in the ventral retina showing the initial uniform random distribution of $10 \%$ of all arbors ( 3000 arbors). $\boldsymbol{B}$, The $\mathrm{N}$-T retinotopic map is overall disorganized, with some retinotopically organized regions (circled). C, The D-V map is globally correct, due to initial order. D, Anterograde tracings show multiple projections with two (left) or three (right, indicated by red arrows) patches aligned along the A-P axis. $E, F$, Ventral N-T/A-P slices showing the evolution of the slice in $A$. The initial random distribution aggregates into layers of arbors (examples shown by red arrows). $\boldsymbol{F}$, The circled layer corresponds to the circled region in $\boldsymbol{A}$ and shows a reverse polarity. $\boldsymbol{G}$, Absence of layers in a dynamic phase displaying no Hebbian plasticity, showing that Hebbian plasticity is the driving force for layer aggregation. $\boldsymbol{H}, \mathrm{N}$-T retinotopic map for $\beta=0$. Without competition, a uniform initial condition leads to collapsed layers of aggregated arbors, and no retinotopic submaps are formed.

based on axons' comparison of EphA levels are incorrect and support the relevance of our model.

\section{Revisiting the function of molecular signaling in retinotopic map development}

We then studied the effect of removing molecular signaling by simulating initial arborizations with random arbor positions along the A-P axis of the SC (Fig. 6A). We observed after the dynamic phase a significant variability in the structure of the resulting mappings. However, several important features were observed consistently and were characteristic of ephrin-A triple knock-out mice (TKO) that lack all retinal and collicular ephrin-As (Pfeiffenberger et al., 2006; Cang et al., 2008). First, the $\mathrm{N}-\mathrm{T}$ retinotopic map was globally disorganized, but some of its parts had an obvious retinotopic organization (Fig. 6A, region circled in black). These "submaps" sometimes displayed a reversed polarity - that is, nasal projections more anterior than temporal ones (as in our example), which has been observed experimentally in TKO mice (Cang et al., 2008). The D-V map, which initially benefited from a more ordered arborization along the L-M axis (see Materials and Methods), had an appropriate retinotopic structure (Fig. $6 \mathrm{C}$ ). Anterograde tracings produced multiple compact axonal patches in the SC, typically two or three with our choice of parameters, aligned along the A-P axis (Fig. $6 \mathrm{D}$, some patches indicated by a red arrow). Over the dynamic phase, the randomly distributed arbors aggregated into compact retinocollicular layers (Fig. 6E, F, red arrows show examples of layers at different stages of their formation; the large circled layer corresponds to the circled submap in Fig. 6B). In our simulations, Hebbian attraction was alone responsible for the formation of layers, as competition did not play any role in it (Fig. 6G). Competition was instead necessary for the formation of submaps because its stretching effect kept layers of aggregated arbors ex- tended along the A-P axis of the SC. Without competition, all axonal projections were concentrated in a few collicular locations along the A-P axis as a result of Hebbian attraction, and no local maps were formed (Fig. $6 H$ ). In summary, a submap arises when the corresponding layer of arbors is extended along the A-P axis and is not "obstructed" by another layer or some of its own portions overlapping the same A-P region. These simulations show that a combination of Hebbian plasticity and axonal competition can instruct local retinotopic order, needlessly of molecular cues, hence explaining occurrences of submaps in mice that lack EphA/ ephrin-A molecular signaling. These simulations also suggest that the function of molecular signaling in WT mice is what plasticity and competition do not alone achieve: creating spatially constrained projections that can be aggregated into a single map and defining the global polarity of the map.

We then focused on the role of reverse signaling by simulating retinocollicular development in mice that lack EphA7 receptors, the most prominent gradient of EphA in the SC (Rashid et al., 2005). In EphA7-KO simulations, we removed reverse signaling during the arborization phase and used for $P_{A}$ the map in Figure $2 B$, which resulted in nasal arbors spanning the whole A-P collicular axis (Fig. $2 F$ ). During the dynamic phase, nasal arbors in the anterior SC aggregated into a compact anterior layer with low arbor density (Fig. $7 A, B$, red arrows) while most other arbors aggregated into a dense WT-like layer (Fig. $7 A, B$, green arrows). A scarce population of nasal axons was not aggregated in layers and subsisted along the A-P axis (Fig. $7 B$, yellow arrows). Simulated anterograde tracings displayed the main EphA7-KO phenotype reported in Rashid et al. (2005). Temporal TZs were essentially normal (Fig. 7C, right panel), and nasal TZs displayed a normal TZ (Fig. 7C, left panel, green arrow) as well as a lowdensity ectopic TZ in the anterior SC (red arrow). Using a lowered maximum in the grayscale of the figure, we were able to 

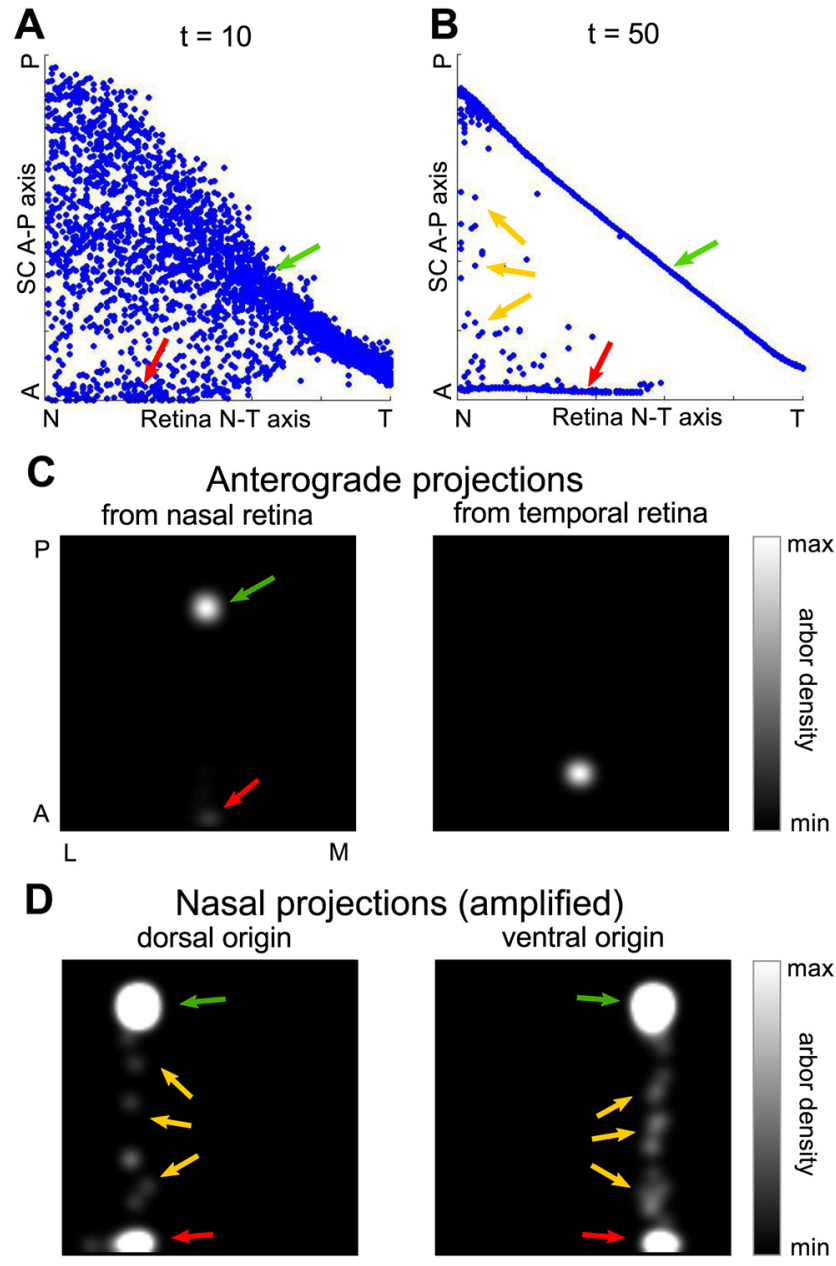

Figure 7. EphA7-K0 simulations. $\boldsymbol{A}, \boldsymbol{B}$, Time course of dynamic phase for initial arborization in Figure 2F. $A, t=10$. A WT-like map (green arrow) and an anterior layer of arbors (red arrow) start to aggregate. $\boldsymbol{B}$, At the end of the dynamic phase, the layers are compact (red and green arrows), and a scarce population of nasal arbors remains, scattered along the A-P axis (yellow arrows). C, Anterograde projections. Nasal projections display a dense TZ at an appropriate retinotopic position in the SC (green arrow) and an anterior low-density ectopic arbor (red arrow). Temporal projections are essentially normal. $\boldsymbol{D}$, Nasal projections from different D-V origins, represented in a color map with a lowered maximum, which amplifies the arbor density signal. The main TZ (green arrow) and the anterior ectopic TZ (red arrow) are clearly visible. Note the two phenomena that have not been experimentally reported: scarce arborizations along the A-P axis (yellow arrows) and significant anterior shifts of the main TZs.

observe the two reported TZs more clearly (Fig. 7D, red and green arrows), together with the scarce arbor population mentioned in Figure $7 B$ (Fig. $7 D$, yellow arrows). We also observed that the main TZ positions were slightly more anterior than in WT (Fig. $7 D$, green arrows). These two additional features remain to be experimentally verified. To our knowledge, this is the first model reproducing EphA7-KO retinocollicular mapping. It suggests that the function of reverse signaling is simply to restrict arborization in the anterior SC to temporal RGCs. This simulation again illustrates the permissive nature of our arborization model, as the final topology of the map is not instructed by molecular signals. It also supports the relevance of this approach. For example, in an instructive chemoaffinity-based model in which arbor positions result from matching gradients of retinal EphA and collicular ephrin-A, there would be no nasal arborizations in the anterior SC, and such a model could thus not explain EphA7-KO phenotypes.
A
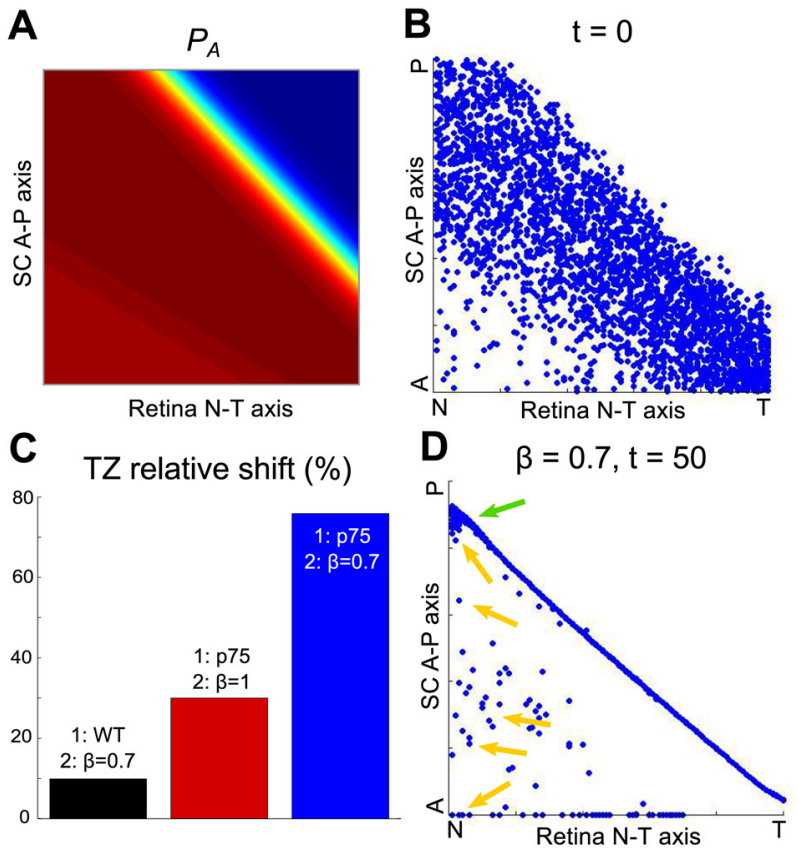

E

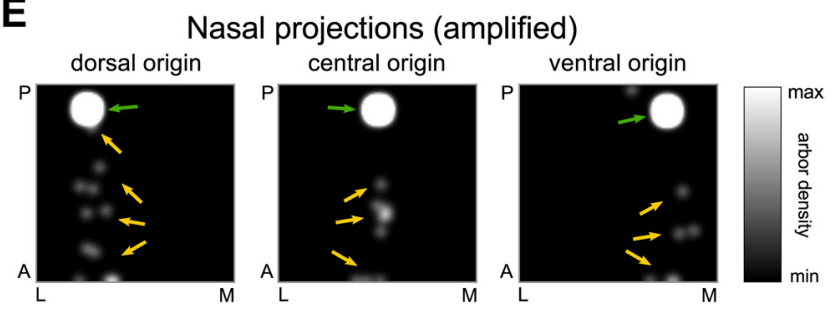

Figure 8. $\quad \mathrm{p} 75^{\mathrm{NTR}}-\mathrm{KO}$ simulations. $A, P_{A}$ map used in the simulation, displaying normal forward signaling and low reverse signaling. $\boldsymbol{B}$, Corresponding arborization. While comparable to a WT arborization, it shows more arbors scattered toward the anterior area of the SC. C, Relative shift of the main TZ of nasal projections in $\mathrm{P} 75^{\mathrm{NTR}}-\mathrm{KO}$ simulations, compared to the average distance between the posterior border of the SC and the TZ centers of WT nasal tracings. "1" labels the type of arborization phase and " 2 " the amplitude of competition in the dynamic phase. Each bar represents an average of over 100 anterograde projections. Reducing both reverse signaling and competition explains the relative shifts observed experimentally (blue bar). Reducing only reverse signaling (red bar) or competition (black bar) did not explain the shifts. $\boldsymbol{D}$, Final state of the mapping, obtained with lowered competition $(\beta=0.7)$ using the arborization in $\boldsymbol{B}$. Nasal arbors show a significant anterior shift (green arrow) compared to their WT counterparts. Scarce arborizations are present, anterior to the main TZ, spanning the A-P axis (yellow arrows). $\boldsymbol{E}$, The main TZ of nasal projections (green arrow) is shifted anteriorly compared to WT, and scarce arbors are present along the A-P axis, mostly anterior to the main TZ (yellow arrows).

Role of $\mathrm{p} 75^{\mathrm{NTR}}$ in reverse signaling and axonal competition p75 neurotrophin receptors play an important part in reverse signaling as coreceptors of retinal ephrin-As and are not involved in forward signaling (Lim et al., 2008). Neonatal p75 ${ }^{\mathrm{NTR}}-\mathrm{KO}$ mice have essentially normal anterograde projections, but at P8 their nasal RGCs have anomalous anterograde tracings. Their main TZ in the posterior SC displays an anterior shift and a few rudimentary branches, and arbors are observed anterior to this TZ along the A-P axis (Lim et al., 2008). We simulated the arborization phase of $\mathrm{p} 75^{\mathrm{NTR}}-\mathrm{KO}$ mice using a $P_{A}$ map in which the inhibitory effect of reverse signaling was drastically reduced (Fig. $8 A$ ). The corresponding arborizations were comparable to WT, but arbors were more scattered in the anterior direction (compare Figs. $8 B, 2 E$ ). Using such initial arborizations, we simulated the dynamic phase with the same parameters as in the WT case, except for the amplitude of competition, $\beta$, that we varied. 
p75 ${ }^{\text {NTR }}$ may indeed be involved in retinocollicular axonal competition, a function that has been shown previously in the sympathetic and olfactory systems (Singh and Miller, 2005; Cao et al., 2007; Singh et al., 2008).

In our simulations, we found that the shifts of nasal axons' main $\mathrm{TZ}$ were best reproduced for $\beta \approx 0.7$. From Lim et al. (2008), we estimated that $\mathrm{p} 75^{\mathrm{NTR}}$-KO main TZ centers lie approximately $75 \%$ further from the posterior collicular border, along the A-P axis, than their WT counterparts. The relative shift increased as $\beta$ decreased, consistent with a lesser "stretching" effect of competition, and for $\beta=0.7$, we found a relative shift of $75.9 \pm 2.1 \%$ (Fig. $8 C$, blue bar, $n=100$ tracings; Fig. $8 D$, $E$, green arrows). Using the same initial arborizations with the WT level of competition $(\beta=1)$, on the other hand, failed to explain the experimentally observed shifts (Fig. $8 C$, red bar). Simulating the dynamic phase with a WT initial condition and $\beta=0.7$ did not explain the anterior shift of the main TZ in nasal tracings (Fig. $8 C$, black bar), so that only the combination of reduced competition and attenuated reverse signaling accounted for this phenotype. Low reverse signaling at the arborization phase allowed a scarce population of nasal arbors to develop in the anterior SC (Fig. 8 B, anteronasal corner), and these remained isolated through the dynamic phase (Fig. 8D,E, yellow arrows). This population was indeed too scarce to aggregate through Hebbian attraction and too isolated to be attracted by the main population of nasal axons in the posterior SC. The biological interpretation of this effect is, hence, that isolated branches generate low activity-dependent plasticity, which is in turn unable to implement their pruning. Their presence in $\mathrm{p} 75^{\mathrm{NTR}}$-KO mice is then not due to a deficit of pruning related to resource-based competition. The second step of the arborization model was crucial in the generation of p75 ${ }^{\text {NTR }}$-KO phenotypes: it eliminated arbors that would otherwise have formed an anterior layer as in EphA7-KO while preserving enough anteriorly scattered arbors.

Our simulations are, to our knowledge, the first to reproduce the retinocollicular development of $\mathrm{p} 75^{\mathrm{NTR}}-\mathrm{KO}$ mice, and we find that reduced competition, together with reduced reverse signaling, is needed to explain the anterior shift of nasal axons' main TZ. Our model hence predicts that $\mathrm{p} 75^{\mathrm{NTR}}$ is involved in retinocollicular axonal competition. Experimental tests of this function could unravel an important mechanism of retinotopic development.

\section{Discussion}

We have developed a new, simple model of retinocollicular development. Our novel permissive arborization process does not rely on the assumption that the retinocollicular map's topology is instructed by molecular signals, and the dynamic phase actively shapes retinotopic order and deeply remodels the map. Our model proposes an alternative to the usual view that retinotopic order is shaped through chemospecificity while activity-dependent processes only serve to refine the map. Within this new framework, we were able to reproduce the main features of retinocollicular development in WT mice as well as several types of transgenic mice. Notably, our simulations lead to several predictions on the nature of axonal competition: Theories based on comparisons of axonal EphA levels are not needed to explain retinocollicular development and could even be proven incorrect experimentally. Instead, we propose that axonal competition is principally based on resource optimization mediated by neurotrophic factors and their receptors, such as p $75^{\text {NTR }}$. Finally, our results clarify the elusive role of reverse signaling by providing the first successful models for EphA7-KO and p $75^{\text {NTR }}$-KO mice.

\section{Permissive vs instructive molecular signaling}

Following Sperry's chemoaffinity hypothesis and the subsequent discovery of retinal and collicular gradients of Ephs and ephrins (Sperry, 1963; Cheng and Flanagan, 1994; Flenniken et al., 1996; Marcus et al., 1996; Zhang et al., 1996), most previous modeling studies have argued that graded molecular cues largely instruct retinotopic map formation (Simpson et al., 2009). Many models have aimed at simulating retinotectal map development as observed in frogs and fish, in which RGC axons enter the tectum through its anterior end and terminate at a roughly correct retinotopic location to arborize. These observations have suggested dynamic models in which molecular cues instruct the position of moving axonal growth cones. In the present study, we focused on mice in which axons, initially overshooting their TZ, span the A-P axis of the SC and arborize selectively along their main shaft. An arborization model based on the more static concept of "interstitial branching" (Yates et al., 2001) was therefore natural. In our model, arborization occurs where total inhibition from independent forward and reverse signaling pathways is relatively lower along the A-P axis of the SC. Surprisingly, this very simple idea had never been explicitly modeled.

Recent models also used interstitial branching, but, contrary to our model, they assumed that gradients of EphA/ephrin-As carry enough information to instruct a coarse map with the same global topology as the final map (Yates et al., 2004; Godfrey et al., 2009). We show here that this seemingly natural assumption is unnecessary to simulate various phenotypes. In our EphA3$\mathrm{KI} / \mathrm{KI}$ simulations, which successfully reproduced the double map phenotype, although all temporal Isl2- axons initially arborize in the anterior SC, as in WT (Fig. $3 H$ ), some of them end up with a $\mathrm{TZ}$ in the middle SC despite their similar EphA/ ephrin-A levels (Fig. 5B, temporal Isl2- arbors). In our simulations of EphA7-KO mice, the final anterior and posterior TZs of nasal axons (Fig. 7C, left) were consistently reproduced, despite the absence of specificity of their arborizations along the A-P axis (Fig. $2 F$ ). In other words, the less instructive nature of our permissive arborization process did not prevent the appearance of appropriate phenotypes and actually allowed the modeling of previously unexplained phenotypes, as in EphA7-KO mice. Importantly, our model makes testable predictions to guide future experiments. For example, anterograde tracings in the temporal retina of EphA3-KI/KI pups or the nasal retina of EphA7-KO pups, and the observation of the corresponding distributions of axons in the SC around age P2-P4, could confirm whether molecular signaling is indeed more permissive than theorized in previous models.

\section{Mechanisms of EphA/ephrin-A molecular signaling}

Our model relies strongly on interstitial branching but remains largely inconclusive on its mechanisms. Our arborization process is not explicitly based on the shape of molecular gradients, so that we are unable to quantify how different concentrations of EphAs and ephrin-As encode the selective inhibition of axonal branching. Moreover, we do not specifically account for EphA/ephrin-A cis-and trans interactions. Indeed, studies in spinal motor and RGC axon development have shown that trans forward signaling between axonal EphA receptors and exogenous ephrin-As in the axon's target is attenuated by cis-interactions with the opposite gradient of endogenous axonal ephrin-As (Hornberger et al., 1999; Carvalho et al., 2006; Kao and Kania, 2011). However, cis-interactions could essentially "sharpen" the net functional gradients of axonal EphAs and ephrin-As available in trans for forward and reverse signaling (Carvalho et al., 2006), so that our 
assumption that these are functionally independent remains valid.

Our permissive arborization phase relies on two principal assumptions. First, initial arborization is achieved at the level of individual RGCs, solely based on collicular cues. Correct retinotectal arborizations in mutant zebrafish larvae displaying only one RGC support this hypothesis (Gosse et al., 2008). Second, axonal positions are not encoded in absolute but in relative levels of low inhibition. This is illustrated in our simulations of ki/ki nasal Isl2 + axons, which develop arbors despite low $P_{A}$ values (Fig. $3 F, H$ ). What kind of branching mechanisms would be compatible with these assumptions? Overshooting axons could integrate the maximum or total level of inhibition by sensing collicular ephrin-As and EphAs along their shaft and could inversely tune their EphA/ephrin-A expression levels, or the level of possible signaling partners, to "normalize" the intensity of molecular inhibition. Sprouts would then occur at A-P positions at which the normalized inhibition is below a certain invariant threshold. Experimental evidence showing the modulation of a gradient of ephrin-A in the goldfish tectum during optic nerve regeneration renders this scenario plausible (Rodger et al., 2000). Alternatively, RGCs' main axonal shaft could contain growing microtubules that continually "push" to form interstitial branches, and these would first sprout where inhibition is lowest, regardless of its absolute level. In this model, the invariant would be the total length of processes produced by axons, which would not depend on the global level of inhibition they encounter. Testing these scenarios would require further experimental and theoretical studies that could help address the critical issue of selective branching.

\section{Competition in retinotopic map development}

Our model proposes that axonal competition favors an optimal occupation of the collicular space, but we specify neither the mechanisms involved nor the object of competition, which could be collicular neurotrophins, available dendritic space allowing synaptic contacts with collicular neurons, or simply physical space for arbors. Brain-derived neurotrophic factor (BDNF) is expressed at a constant level across the SC and could be a limiting factor sensed by RGCs competing for space (Cohen-Cory and Fraser, 1995; Marler et al., 2008). Whether neurotrophins are the object of competition, however, remains an open question. Several studies have shown that electric activity in axons mediates competitive interactions leading to arbor changes by selective axonal growth, pruning, and synaptic plasticity (Cohen-Cory and Lom, 2004; Hua et al., 2005; Ben Fredj et al., 2010). Some of them demonstrated the involvement of neurotrophins and their receptors in these processes, in particular $\mathrm{p} 75^{\mathrm{NTR}}$, which mediates competitive axon pruning upon binding BDNF (Singh and Miller, 2005; Cao et al., 2007; Singh et al., 2008). This mechanism could function in the competitive optimization of collicular space by $75^{\text {NTR }}$ predicted by our model, which remains to be demonstrated experimentally and could benefit from more specific models of neurotrophic signaling.

Reber et al. (2004) propose a competition mechanism based on comparisons of axonal EphA levels, explaining the formation of a double map in EphA3-KI/KI mice. This model predicts that the anterior and posterior maps are, respectively, composed of Isl2 + and Isl2- axons. This theory could be tested by making retrograde tracings from the anterior part of the SC of EphA3$\mathrm{KI} / \mathrm{KI}$ mice and testing the genotype of back-traced RGCs. Contrary to that of Reber et al. (2004), our model predicts that axonal projections in the anterior SC belong to both Isl2- and Isl2+ temporal axons (Fig. $5 B, F$ ). The verification of this prediction would strongly hint at whether competition based on comparisons of axonal molecular cue levels is a valid theory. Nevertheless, the proportion of Isl2- axonal processes in the anterior map predicted by our model should not be regarded as a quantitative prediction because changes in model parameters can significantly affect the curve in Figure 5F. If our prediction is proven incorrect, it would suggest that the theory of EphA-based competition could be valid but would require further experimental validation. Alternatively, temporal Isl2 - arbors could be eliminated from the anterior SC by another yet uncovered mechanism.

In conclusion, our novel modeling approach based on permissive arborization accounted for complex phenotypes that had been observed experimentally but not simulated by previous models. Moreover, we were able to formulate several testable predictions, which was rarely done in previous modeling studies in the field. Testing them could significantly advance our understanding of topographic map development.

\section{References}

Ben Fredj N, Hammond S, Otsuna H, Chien CB, Burrone J, Meyer MP (2010) Synaptic activity and activity-dependent competition regulates axon arbor maturation, growth arrest, and territory in the retinotectal projection. J Neurosci 30:10939-10951.

Brown A, Yates PA, Burrola P, Ortuño D, Vaidya A, Jessell TM, Pfaff SL, O'Leary DD, Lemke G (2000) Topographic mapping from the retina to the midbrain is controlled by relative but not absolute levels of EphA receptor signaling. Cell 102:77-88.

Butts DA, Rokhsar DS (2001) The information content of spontaneous retinal waves. J Neurosci 21:961-973.

Cang J, Wang L, Stryker MP, Feldheim DA (2008) Roles of ephrin-As and structured activity in the development of functional maps in the superior colliculus. J Neurosci 28:11015-11023.

Cao L, Dhilla A, Mukai J, Blazeski R, Lodovichi C, Mason CA, Gogos JA (2007) Genetic modulation of bdnf signaling affects the outcome of axonal competition in vivo. Curr Biol 17:911-921.

Carvalho RF, Beutler M, Marler KJ, Knöll B, Becker-Barroso E, Heintzmann R, Ng T, Drescher U (2006) Silencing of epha3 through a cis interaction with ephrina5. Nat Neurosci 9:322-330.

Cheng HJ, Flanagan JG (1994) Identification and cloning of ELF-1, a developmentally expressed ligand for the Mek4 and Sek receptor tyrosine kinases. Cell 79:157-168.

Chklovskii DB, Koulakov AA (2004) Maps in the brain: what can we learn from them? Annu Rev Neurosci 27:369-392.

Cohen-Cory S, Fraser SE (1995) Effects of brain-derived neurotrophic factor on optic axon branching and remodelling in vivo. Nature 378:192-196.

Cohen-Cory S, Lom B (2004) Neurotrophic regulation of retinal ganglion cell synaptic connectivity: from axons and dendrites to synapses. Int J Dev Biol 48:947-956.

Dhande OS, Hua EW, Guh E, Yeh J, Bhatt S, Zhang Y, Ruthazer ES, Feller MB, Crair MC (2011) Development of single retinofugal axon arbors in normal and $\beta 2$ knock-out mice. J Neurosci 31:3384-3399.

Feldheim DA, Vanderhaeghen P, Hansen MJ, Frisén J, Lu Q, Barbacid M, Flanagan JG (1998) Topographic guidance labels in a sensory projection to the forebrain. Neuron 21:1303-1313.

Flenniken AM, Gale NW, Yancopoulos GD, Wilkinson DG (1996) Distinct and overlapping expression patterns of ligands for Eph-related receptor tyrosine kinases during mouse embryogenesis. Dev Biol 179:382-401.

Frisén J, Yates PA, McLaughlin T, Friedman GC, O’Leary DD, Barbacid M (1998) Ephrin-A5 (AL- 1/RAGS) is essential for proper retinal axon guidance and topographic mapping in the mammalian visual system. Neuron 20:235-243.

Galli L, Maffei L (1988) Spontaneous impulse activity of rat retinal ganglion cells in prenatal life. Science 242:90-91.

Godfrey KB, Eglen SJ, Swindale NV (2009) A multi-component model of the developing retinocollicular pathway incorporating axonal and synaptic growth. PLoS Comput Biol 5:e1000600.

Goodhill GJ, Xu J (2005) The development of retinotectal maps: a review of models based on molecular gradients. Network 16:5-34. 
Goodman CS, Shatz CJ (1993) Developmental mechanisms that generate precise patterns of neuronal connectivity. Cell 72(Suppl):77-98.

Gosse NJ, Nevin LM, Baier H (2008) Retinotopic order in the absence of axon competition. Nature 452:892-895.

Hebb DO (1949) The organization of behavior: a neurophysiological theory. New York: Wiley.

Hindges R, McLaughlin T, Genoud N, Henkemeyer M, O’Leary DD (2002) $\mathrm{EphB}$ forward signaling controls directional branch extension and arborization required for dorsal-ventral retinotopic mapping. Neuron 35:475-487.

Hornberger MR, Dütting D, Ciossek T, Yamada T, Handwerker C, Lang S, Weth F, Huf J, Wessel R, Logan C, Tanaka H, Drescher U (1999) Modulation of epha receptor function by coexpressed ephrin-A ligands on retinal ganglion cell axons. Neuron 22:731-742.

Hua JY, Smear MC, Baier H, Smith SJ (2005) Regulation of axon growth in vivo by activity-based competition. Nature 434:1022-1026.

Kalatsky VA, Stryker MP (2003) New paradigm for optical imaging: temporally encoded maps of intrinsic signal. Neuron 38:529-545.

Kao TJ, Kania A (2011) Ephrin-mediated cis-attenuation of Eph receptor signaling is essential for spinal motor axon guidance. Neuron 71:76-91.

Lim YS, McLaughlin T, Sung TC, Santiago A, Lee KF, O'Leary DD (2008) p75(NTR) mediates ephrin-A reverse signaling required for axon repulsion and mapping. Neuron 59:746-758.

Marcus RC, Gale NW, Morrison ME, Mason CA, Yancopoulos GD (1996) Eph family receptors and their ligands distribute in opposing gradients in the developing mouse retina. Dev Biol 180:786-789.

Marler KJ, Becker-Barroso E, Martínez A, Llovera M, Wentzel C, Poopalasundaram S, Hindges R, Soriano E, Comella J, Drescher U (2008) A TrkB/EphrinA interaction controls retinal axon branching and synaptogenesis. J Neurosci 28:12700-12712.

McLaughlin T, O'Leary DD (2005) Molecular gradients and development of retinotopic maps. Annu Rev Neurosci 28:327-355.

McLaughlin T, Torborg CL, Feller MB, O’Leary DD (2003) Retinotopic map refinement requires spontaneous retinal waves during a brief critical period of development. Neuron 40:1147-1160.

Meister M, Wong RO, Baylor DA, Shatz CJ (1991) Synchronous bursts of action potentials in ganglion cells of the developing mammalian retina. Science 252:939-943.

Pfeiffenberger C, Yamada J, Feldheim DA (2006) Ephrin-As and patterned retinal activity act together in the development of topographic maps in the primary visual system. J Neurosci 26:12873-12884.

Rashid T, Upton AL, Blentic A, Ciossek T, Knöll B, Thompson ID, Drescher U (2005) Opposing gradients of ephrin-As and EphA7 in the superior colliculus are essential for topographic mapping in the mammalian visual system. Neuron 47:57-69.

Reber M, Burrola P, Lemke G (2004) A relative signalling model for the formation of a topographic neural map. Nature 431:847-853.
Rodger J, Bartlett CA, Beazley LD, Dunlop SA (2000) Transient upregulation of the rostrocaudal gradient of ephrin-A2 in the tectum coincides with reestablishment of orderly projections during optic nerve regeneration in goldfish. Exp Neurol 166:196-200.

Ruthazer ES, Cline HT (2004) Insights into activity-dependent map formation from the retinotectal system: a middle-of-the-brain perspective. J Neurobiol 59:134-146.

Ruthazer ES, Akerman CJ, Cline HT (2003) Control of axon branch dynamics by correlated activity in vivo. Science 301:66-70.

Simpson HD, Mortimer D, Goodhill GJ (2009) Theoretical models of neural circuit development. Curr Top Dev Biol 87:1-51.

Singh KK, Miller FD (2005) Activity regulates positive and negative neurotrophin-derived signals to determine axon competition. Neuron 45:837-845.

Singh KK, Park KJ, Hong EJ, Kramer BM, Greenberg ME, Kaplan DR, Miller FD (2008) Developmental axon pruning mediated by bdnf-p75ntrdependent axon degeneration. Nat Neurosci 11:649-658.

Sperry RW (1963) Chemoaffinity in the orderly growth of nerve fiber patterns and connections. Proc Natl Acad Sci U S A 50:703-710.

Stafford BK, Sher A, Litke AM, Feldheim DA (2009) Spatial-temporal patterns of retinal waves underlying activity-dependent refinement of retinofugal projections. Neuron 64:200-212.

Triplett JW, Owens MT, Yamada J, Lemke G, Cang J, Stryker MP, Feldheim DA (2009) Retinal input instructs alignment of visual topographic maps. Cell 139:175-185.

Triplett JW, Pfeiffenberger C, Yamada J, Stafford BK, Sweeney NT, Litke AM, Sher A, Koulakov AA, Feldheim DA (2011) Competition is a driving force in topographic mapping. Proc Natl Acad Sci U S A 108:19060-19065.

Tsigankov DN, Koulakov AA (2006) A unifying model for activitydependent and activity-independent mechanisms predicts complete structure of topographic maps in ephrin-A deficient mice. J Comput Neurosci 21:101-114

Udin SB, Gaze RM (1983) Expansion and retinotopic order in the goldfish retinotectal map after large retinal lesions. Exp Brain Res 50:347-352.

Yates PA, Roskies AL, McLaughlin T, O'Leary DD (2001) Topographicspecific axon branching controlled by ephrin-As is the critical event in retinotectal map development. J Neurosci 21:8548-8563.

Yates PA, Holub AD, McLaughlin T, Sejnowski TJ, O'Leary DD (2004) Computational modeling of retinotopic map development to define contributions of EphA-ephrinA gradients, axon-axon interactions, and patterned activity. J Neurobiol 59:95-113.

Zhang JH, Cerretti DP, Yu T, Flanagan JG, Zhou R (1996) Detection of ligands in regions anatomically connected to neurons expressing the Eph receptor Bsk: potential roles in neuron-target interaction. J Neurosci 16: 7182-7192. 